Arabian Journal of Science and Engineering

ISSN: 2193-567X (print version); ISSN: 2191-4281 (electronic version)

Publisher- Springer; Impact factor $=1.092$

ACCEPTED MAY 20 2019

\title{
TRANSIENT ANALYSIS OF THIRD-GRADE VISCOELASTIC NANOFLUID FLOW EXTERNAL TO A HEATED CYLINDER WITH BUOYANCY EFFECTS
}

\author{
Ashwini Hiremath ${ }^{1}$, G. Janardhana Reddy ${ }^{1,}$ * and O. Anwar Bég ${ }^{2}$ \\ ${ }^{1}$ Department of Mathematics, Central University of Karnataka, Kalaburagi, India. \\ ${ }^{2}$ Fluid Mechanics, Aeronautical and Mechanical Engineering Department, School of \\ Computing, Science and Engineering, University of Salford, Manchester M54WT, UK.
}

*Corresponding author-Email: gjr@cuk.ac.in

\begin{abstract}
Nanotechnology is rapidly embracing numerous areas of manufacturing and process engineering. New types of nanomaterials are being exploited to improve, for example, coating integrity, anti-corrosion characteristics and other features of fabricated components. Motivated by these developments, in the current study a mathematical model is developed for unsteady free-convective laminar flow of third-grade viscoelastic fluid (doped with nano-particles) from a semi-infinite vertical isothermal cylinder, as a model of thermal coating flow of a pipe geometry. Non-Newtonian behavior is simulated with the thermodynamically robust third grade Reiner-Rivlin model which accurately represents polymer fluids. Nanoscale effects are analyzed with the Buongiorno two-component nanofluid model. The governing equations comprise a set of highly coupled, nonlinear, multi-degree partial differential equations featuring viscoelastic and nanofluid parameters. An implicit Crank-Nicolson numerical scheme is implemented to solve the emerging nonlinear problem with appropriate initial and boundary conditions. Detailed graphical plots for velocity, temperature and nano-particle volume fraction are presented for a range of different parameters (i.e., third-grade fluid parameter, Brownian motion parameter, thermophoretic parameter, buoyancy ratio parameter, Lewis number). Additionally, distributions of the heat transfer coefficient, skin friction and Sherwood number at the cylinder surface are visualized. Furthermore, streamlines, isotherms and nano-particle volume fraction contour plots are included for variation of the third-grade parameter. Contour plots for the third-grade nanofluid flow are found to deviate significantly from those corresponding to Newtonian nanofluids. Validation of the numerical solutions with earlier studies is also included.
\end{abstract}

KEYWORDS: Third-grade viscoelastic nanofluid, Thermal convection, Cylinder, Thermophoresis, Brownian motion, Implicit numerical method, Contour plots, Industrial coating.

\section{NOMENCLATURE}

$\mathrm{g}^{\prime} \quad$ acceleration due to gravity 
Gr Grashof number

$\operatorname{Pr} \quad$ Prandtl number

$C_{p} \quad$ specific heat at constant pressure

Sh Sherwood number

$\bar{C}_{f} \quad$ dimensionless average momentum transport coefficient

$\overline{\mathrm{Nu}} \quad$ average heat transport coefficient

$k \quad$ thermal conductivity

$r_{o} \quad$ radius of the cylinder

$t \quad$ dimensionless time

$t^{\prime} \quad$ time

$P \quad$ fluid pressure

$T^{\prime} \quad$ temperature

$D_{B} \quad$ coefficient of Brownian diffusion

$D_{T} \quad$ coefficient of thermophoresis diffusion

T dimensionless temperature

$t r \quad$ trace

$x, r \quad$ axial and radial coordinates, respectively

$u, v \quad$ velocity components in $(x, r)$ coordinate system

$X, R \quad$ dimensionless axial and radial coordinate

$U, V$ dimensionless velocity components in $X, R$ directions, respectively

$\mathrm{Nr} \quad$ buoyancy ratio parameter

$\mathrm{Nb} \quad$ Brownian motion parameter

Le Lewis number

$N t \quad$ thermophoretic parameter 


\section{Greek letters}

$\beta_{T} \quad$ volumetric thermal expansion coefficient

$\alpha \quad$ thermal diffusivity

$\beta \quad$ non-dimensional third-grade fluid parameter

$\rho_{f} \quad$ density of base fluid (i.e., third-grade fluid)

$\rho_{p} \quad$ density of nanoparticles

$\psi \quad$ stream function

$\varphi \quad$ dimensional volume fraction

$\mu \quad$ viscosity of the nanofluid

$\Theta \quad$ dimensionless volume fraction (nano-particle species concentration)

$\vartheta \quad$ kinematic viscosity

\section{Subscripts}

$f, g \quad$ grid levels in $(X, R)$ coordinate system

w wall conditions

$\infty \quad$ ambient conditions

\section{Superscript}

$h \quad$ time level

\section{INTRODUCTION}

Nanomaterials are rapidly infiltrating into diverse applications in modern technology. They include nano-composites [1], nano-polymers [2], nano-films [3] and many others. Nanomaterials are engineered at the nanoscale and feature embedding of nano-structures 
(nano-shells, nano-particles, nano-wires etc) in materials. In coating applications, nanopolymers, which are nanofluids [4] comprising nano-particles suspended in polymer base fluids, have been shown to achieve improved resistance and durability compared with conventional coatings. Important studies in for example tribological nano-coatings include [5]. In thermal processing of nano-polymers for coating [6] for example, higher thermal conductivity is advantageous. Regular coating materials e.g. conventional polymers and also other heat transfer fluids (water, engine oil, ethylene glycol) are relatively inefficient thermally. Masuda et al. [7] demonstrated the increment in thermal efficiency via suspending ultra-fine solid particles in a regular fluid since solid particles (in particular metallic ones) have better thermal conductivities than liquids. They also showed that ultra-fine submicro-sized (nano) particles attain superior performance to conventional microscopic particles. Due to their extremely small sizes, nano-particles reduce clogging and provide a larger surface area for heat transfer. Nanofluids may be synthesized via produced by dispersing the nano-sized materials such as nanoparticles, nanosheet, nanotubes, nanofibers, nanowires, nanorods in regular ("base") fluids. A nano-coating supplies more surface area, minimum surface energy, greater thermal conductivity, higher durability, more sustainability (it is more "green") and is hydrophobic in nature. The performance of for example aerospace landing gear components, gas turbine blades, and heat exchangers can be enhanced using thermal nano-coating which increases the wear resistance, dissipates heating and extends the life of coatings and components via mitigation of oxidation, corrosion, abrasion and swarfing. Nano-coatings also add high hydrophobicity to the geometrical surface in direct contact with the fluid by elevating heat transfer performance and minimizing energy losses due to friction. Nano-coatings therefore impart significant tribological, coating and sustainable properties to working surfaces. Xuan and Li [8] have used the hot wire method to show that thermal conductivity increases with greater volume fraction of nanoparticles. Xuan and Roetzel [9] have presented 
a model for dispersion of thermal energy in nanofluids. Putra et al. [10] have investigated the effects of material type (silicon carbide, titanium oxide, zinc oxide etc), concentration of nanoparticles and also enclosure geometry on nanofluid performance. Timofeeva et al. [11] have studied the impact of different nanoscale parameters on the enhancement of thermal conductivity. Yu and Xie [12] elaborated in detail on the stability and synthesis of robust nanofluids. In parallel with these experimental studies, considerable progress in nanofluid mathematical modelling has also been made. Buongiorno [13] proposed a two-component homogenous model to discuss different slip mechanisms characterizing thermal convection in nanofluids in which Brownian motion and thermophoresis were identified as the key mechanisms for heat transfer enhancement. In this model momentum, energy and nano-particle species conservation are all considered. The alternative Tiwari-Das model [14] features only momentum and energy conservation equations although it does allow nano-particle volume fraction to be varied and also different nano-particle properties to be studied. Both models have been recently reviewed in spin coating and other engineering applications by Bég [15]. Buongiorno's model however accommodates comfortably boundary-layer flows and has therefore been adopted in numerous analytical and computational studies of nanofluid transport in recent years. These include free convection boundary layer flows [16], time-dependent wavy enclosure flows [17], transient stretching sheet dynamics [18], high-temperature magnetized coating flows of cylinders [19], entropy generation towards heated surface [20], enrobing flows of spherical bodies [21], spin coating of spherical containers [22] and unsteady free convection boundary layers from an upright cylinder [23].

The above studies have considered the nanofluid to be Newtonian. However, the classical fluid theory does not explain many characteristics of nanofluids fluids such as threshold stress, nonlinearity of creeping, relaxation of stress, normal stress differences, shear thickening/thinning, temperature-dependent viscosity and memory. Numerous experimental studies have 
established that nanofluids exhibit strong non-Newtonian characteristics largely attributable to the presence of nano-particles which modify the shear stress-strain nature of the base fluid. For example, in coating systems, the nanofluid will experience large deformation or continous shearing. Nano-silica agglomeration may manifest in the form of a gel network in heated nanocoatings during enrobing processes [24]. Viscoelastic and viscoplastic behaviour has also been observed in nanofluids in thermal polymer processing, as noted by Aoki et al. [25] (for carbon black nano-suspensions in polymers), Du et al. [26] (for polymer nanocomposite fillers and surface deposition materials), Elias et al. [27] (in immiscible polymer blends stabilized with nano-silica particles), and Chang et al. [28] (in copper oxide polymer-based nanofluids). To properly simulate the thermofluid dynamics of real nanofluids it is therefore judicious to utilize an appropriate non-Newtonian theory (i.e., the extension of classical fluid theory) coupled with the nanoscale model. Many elegant formulations exist for rheological modelling including rate type, integral type and differential type formulations which robustly capture the non-linear stress-strain characteristic of nanofluids. In recent years a diverse spectrum of such theories has been deployed to analyze rheological behavior of nanofluids in many different systems including coating dynamics and solar pumps. These include the Eringen micropolar model [29], Williamson elastic-viscous model [30], Ostwald-DeWaele power-law model (pseudoplastic and dilatant nanofluids) [31], second-order viscoelastic Reiner-Rivlin model [32], Casson viscoplastic (yield stress) models [33] and Reynolds exponential viscosity model [34]. The differential type fluid model (Reiner-Rivlin family of models) lucidly simulates the relationship between the stress history and deformation gradient which are significant in real polymer flows. One of the higher order classes of differential type is the third-grade fluid model. It can elucidate shear thinning (decrease in shear viscosity with increasing shear rate) or shear thickening (elevation in shear viscosity with increasing shear rate) behavior of polymers [35], [36] and features additional rheological material parameters compared with 
other viscoelastic models such as the second-order model, Maxwell model or Oldroyd-B model. In addition to polymers, the third- grade model also quite accurately models the nonNewtonian behavior of certain coolants and lubricants, slurries and biological liquids. Many heat transfer studies have featured the third-grade model including mixed convection stagnation flows [37], viscoelastic thermal convection in porous media [38], Hiemenz flow with thermal convection [39], boundary layer slip convection from an expanding sheet [40], magnetohydrodynamic (MHD) flow of unsteady convective third-grade fluid in a cylindrical system [41], time-dependent thermal convection from an upright cylinder [42], supercritical third-grade fluid heat transfer external to a vertical cylinder [43] and very recently the hydromagnetic convection from an isothermal inverted with radiative flux [44]. A number of investigations have also addressed third-grade viscoelastic nanofluid flows. Farooq et al. [45] used an analytical approach to investigate buoyancy-driven two-layer channel flows of a thirdgrade nanofluid. Nadeem and Saleem [46] considered boundary layer convection of third-grade nanofluids from a spinning cone. Khan et al. [47] employed the Buongiorno nanoscale and third-grade fluid models to simulate the transport phenomena from a convectively-heated stretching permeable surface. Qayyum et al. [48] reported on radiative flux and heat source/sink effects in hydromagnetic third-grade nanofluid flow. Hayat et al. [49] presented analytical solutions for chemically-reacting third-grade nanofluid from a rotating stretchable disk. These studies all confirmed the significant deviation of third-grade non-Newtonian thermal/flow characteristics from Newtonian results.

In the present article, a theoretical investigation is conducted for time-dependent natural convection boundary layer flow of third-grade viscoelastic nanofluid external to a semi-infinite vertical isothermal cylinder. To the authors' knowledge this problem which is of relevance in thermal nano-coating enrobing flows, has not yet been addressed in the literature. NonNewtonian behavior is simulated with the thermodynamically robust third-grade Reiner-Rivlin 
model which accurately represents polymer fluids. Nanoscale effects are analyzed with the Buongiorno two-component nanofluid model. The non-dimensional flow model is solved under suitable initial and boundary conditions with the implicit Crank-Nicolson numerical scheme. Validation of the code with earlier Newtonian nanofluid simulations [e.g. Chamkha et al. [23]]. Graphical visualization of steady-state velocity, temperature and nano-particle volume fraction distributions are presented for a range of different parameters (i.e., third-grade fluid parameter, Brownian motion parameter, thermophoretic parameter, buoyancy ratio parameter, Lewis number). Additionally, distributions of the heat transfer coefficient, skin friction, Sherwood number, streamlines, isotherms and nano-particle volume fraction contours are included for variation of the third-grade parameter.

\section{NANOFLUID VISCOELASTIC FLOW MODEL}

The problem under consideration comprises the unsteady boundary layer flow of an incompressible, non-Newtonian (third-grade viscoelastic) nanofluid from a uniformly heated semi-infinite stationary vertical cylinder, as depicted in Fig. 1.

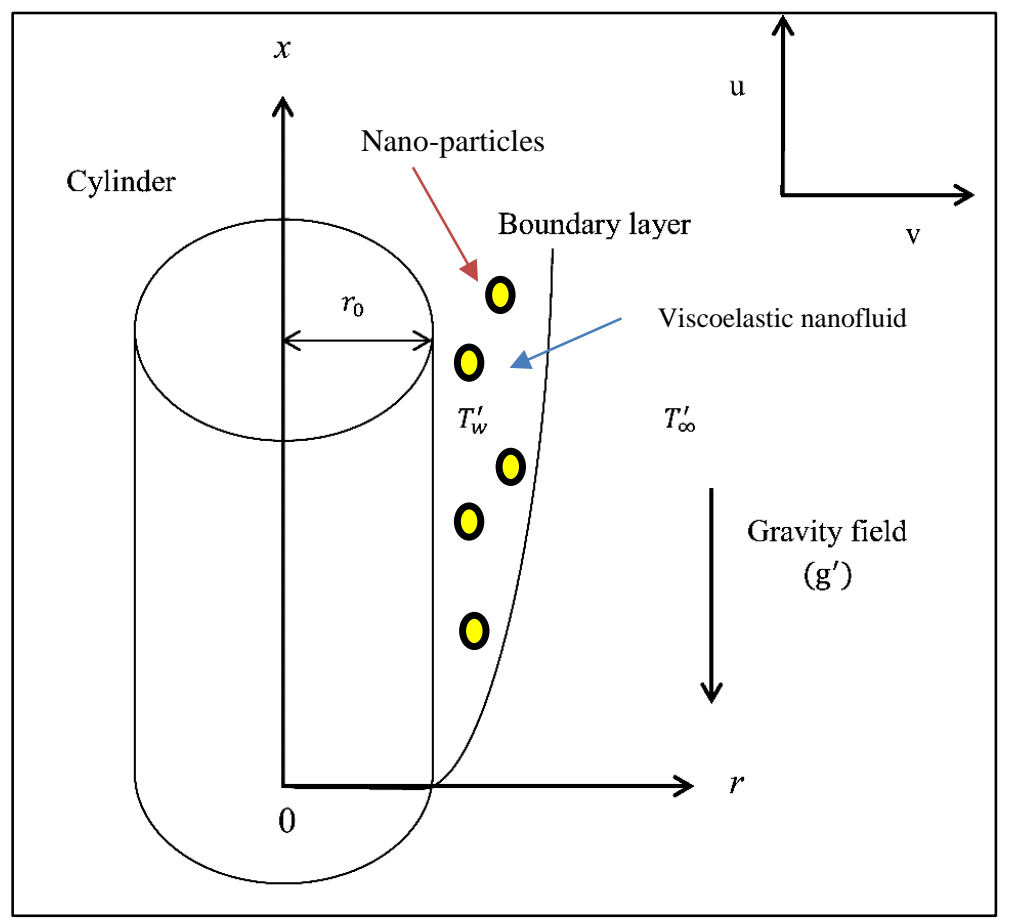


Fig. 1. Physical model for nano-polymer coating flow on a cylinder.

A rectangular coordinate system describes the flow-domain, i.e., $x$-axis (axial coordinate) is orientated along the cylinder, and the $r$-axis (radial axis) is directed normal to the cylinder longitudinal axis. At the initiation of flow (i.e., at $t^{\prime}=0$ ), the cylinder and third-grade nanofluid are maintained at the same temperature (i.e., at $T_{\infty}^{\prime}$ ). A temperature difference is sustained with progress in time $\left(t^{\prime}>0\right)$, so that cylinder surface temperature is enhanced to $T_{w}^{\prime}$ and this constant temperature is preserved at all subsequent times.

\subsection{Formation of constitutive relation for third-grade fluid model:}

The constitutive relation defined by Cauchy's stress-tensor $\left(\tau^{\prime}\right)$ for third-grade viscoelastic fluids with thermodynamic compatibility (Fosdick and Rajagopal [35]) is as follows:

$\tau^{\prime}=-P I+\mu S_{1}^{\prime}+\alpha_{1}^{\prime} S_{2}^{\prime}+\alpha_{2}^{\prime} S_{1}^{\prime 2}+\beta_{1}^{\prime} S_{3}^{\prime}+\beta_{2}^{\prime}\left(S_{1}^{\prime} S_{2}^{\prime}+S_{2}^{\prime} S_{1}^{\prime}\right)+\beta_{3}^{\prime}\left(\operatorname{tr} S_{1}^{\prime 2}\right) S_{1}^{\prime}$

Here $\alpha_{l}^{\prime}(l=1,2)$ and $\beta_{l}^{\prime}(l=1,2,3)$ are material constants depending on temperature variations, $-P I$ refers to the spherical part of stress-tensor, and $S_{l}^{\prime}(l=1,2,3)$ signify the Rivlin-Ericksen tensor matrices which are given through the equations:

$S_{1}^{\prime}=(\nabla \boldsymbol{V})^{T^{* *}}+\nabla \boldsymbol{V}, S_{l}^{\prime}=\frac{d S_{l-1}^{\prime}}{d t}+(\nabla \boldsymbol{V})^{T^{* *}} S_{l-1}^{\prime}+S_{l-1}^{\prime}(\nabla \boldsymbol{V}), \quad l=2,3 \ldots$

where $T^{* *}, \boldsymbol{V}, \nabla$ correspond to the matrix transposition, velocity vector, gradient operator,

respectively. $\frac{d}{d t}$ denotes the material derivative which is expressed as $\frac{d}{d t}(\cdot)=\left(\frac{\partial}{\partial t}+V \nabla\right)(\cdot)$.

Also, for the third-grade fluid model it is necessary to satisfy the following assumptions, i.e.,

(i) 'Clausius-Duhem' inequality.

(ii) least value of 'Helmholtz free-energy' at equilibrium. 
The restrictions on third-grade fluid parameters satisfying the above assumptions are given by the following constraints,

$\mu \geq 0 ; \quad \alpha_{1}^{\prime} \geq 0 ; \quad\left|\alpha_{1}^{\prime}+\alpha_{2}^{\prime}\right| \leq \sqrt{24 \mu \beta_{3}^{\prime}}$

$\beta_{1}^{\prime}=0 ; \quad \beta_{2}^{\prime}=0 ; \quad \beta_{3}^{\prime} \geq 0$

Further, fluid thermodynamics, boundedness and stability criteria are shown similar to secondgrade fluid (scrutinized by Dunn and Fosdick [50], for $\beta_{3}^{\prime}=0$, it provides constitutive relation for second-grade fluid). Fosdick and Straughan [51] showed that non-physical results can be induced for $\alpha_{1}^{\prime}<0$ and asymptotic stability condition for $\alpha_{1}^{\prime} \geq 0$.

Substitution in Eqn. (1), leads to:

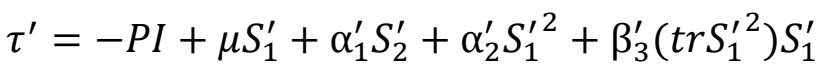

$\tau^{\prime}=-P I+S$

Here $S$ is the extra stress-tensor i.e.,

$$
\begin{aligned}
& S=\mu \frac{1}{r} \frac{\partial}{\partial r}\left(r \frac{\partial u}{\partial r}\right)+\alpha_{1}^{\prime}\left[\frac{1}{r} \frac{\partial^{2} u}{\partial r \partial t^{\prime}}+\frac{\partial^{3} u}{\partial r^{2} \partial t^{\prime}}+v \frac{\partial^{3} u}{\partial r^{3}}+2 \frac{\partial v}{\partial r} \frac{\partial^{2} u}{\partial r^{2}}+\frac{\partial u}{\partial r} \frac{\partial^{2} v}{\partial r^{2}}+3 \frac{\partial^{2} u}{\partial r^{2}} \frac{\partial u}{\partial x}+4 \frac{\partial u}{\partial r} \frac{\partial^{2} u}{\partial x \partial r}\right. \\
& \left.+\frac{v}{r} \frac{\partial^{2} u}{\partial r^{2}}+\frac{3}{r} \frac{\partial u}{\partial x} \frac{\partial u}{\partial r}+\frac{u}{r} \frac{\partial^{2} u}{\partial x \partial r}+u \frac{\partial^{3} u}{\partial x \partial r^{2}}+\frac{1}{r} \frac{\partial u}{\partial r} \frac{\partial v}{\partial r}\right]+\alpha_{2}^{\prime}\left[\frac{2}{r} \frac{\partial u}{\partial r} \frac{\partial v}{\partial r}+2 \frac{\partial^{2} u}{\partial r^{2}} \frac{\partial u}{\partial x}+\frac{2}{r} \frac{\partial u}{\partial x} \frac{\partial u}{\partial r}+\right. \\
& \left.2 \frac{\partial^{2} v}{\partial r^{2}} \frac{\partial u}{\partial r}+4 \frac{\partial u}{\partial r} \frac{\partial^{2} u}{\partial x \partial r}+2 \frac{\partial v}{\partial r} \frac{\partial^{2} u}{\partial r^{2}}\right]+\beta_{1}^{\prime}\left[\frac{2}{r}\left(\frac{\partial u}{\partial r}\right)^{3}+\left(\frac{\partial u}{\partial r}\right)^{2}\left(6 \frac{\partial^{2} u}{\partial r^{2}}+4 \frac{\partial^{2} u}{\partial x^{2}}\right)+2 \frac{\partial^{2} u}{\partial x \partial r} \frac{\partial u}{\partial x} \frac{\partial u}{\partial r}\right]
\end{aligned}
$$

where the velocity components $u, v$ are taken along $x, r$ - directions, respectively.

\subsection{Flow analysis:}

The conservation equations for buoyancy-induced free-convective flow of third-grade nanofluid are based on all assumptions and standard definitions as mentioned earlier including 
the Boussinesq approximation $[52,53]$. The axisymmetric viscoelastic nanofluid thermal convection from the curved geometry (cylinder) is represented by the following momentum, energy and nano-particle species conservation equations featuring Brownian and thermophoresis effects $[42,43,49,54,55,56]$ :

$$
\begin{aligned}
& \frac{\partial(r u)}{\partial x}+\frac{\partial(r v)}{\partial r}=0 \\
& \frac{\partial P}{\partial x}=-\rho_{f}\left(u \frac{\partial u}{\partial x}+v \frac{\partial u}{\partial r}+\frac{\partial u}{\partial t^{\prime}}\right)+\mathrm{g}^{\prime}\left[\left(1-\varphi_{\infty}\right) \rho_{f_{\infty}} \beta_{T}\left(T^{\prime}-T_{\infty}^{\prime}\right)-\left(\rho_{p}-\rho_{f_{\infty}}\right)\left(\varphi-\varphi_{\infty}\right)\right]+S
\end{aligned}
$$

$\frac{\partial P}{\partial r}=0$

$u \frac{\partial T^{\prime}}{\partial x}+v \frac{\partial T^{\prime}}{\partial r}+\frac{\partial T^{\prime}}{\partial t^{\prime}}=\frac{\alpha}{r} \frac{\partial}{\partial r}\left(r \frac{\partial T^{\prime}}{\partial r}\right)+\omega\left[D_{B} \frac{\partial \varphi}{\partial r} \frac{\partial T^{\prime}}{\partial r}+\frac{D_{T}}{T_{\infty}}\left(\frac{\partial T}{\partial r}\right)^{2}\right]$

$u \frac{\partial \varphi}{\partial x}+v \frac{\partial \varphi}{\partial r}+\frac{\partial \varphi}{\partial t^{\prime}}=D_{B} \frac{1}{r} \frac{\partial}{\partial r}\left(r \frac{\partial \varphi}{\partial r}\right)+\frac{D_{T}}{T_{\infty}} \frac{1}{r} \frac{\partial}{\partial r}\left(r \frac{\partial T^{\prime}}{\partial r}\right)$

The relevant initial and boundary conditions are imposed as:

$$
\begin{array}{rlrl}
t^{\prime} \leq 0: & T^{\prime}=T_{\infty}^{\prime}, \quad v=0, \varphi=\varphi_{\infty}, & u=0 & \text { for all } x \text { and } r \\
t^{\prime}>0: & T^{\prime}=T_{w}^{\prime}, \quad v=0, \varphi=\varphi_{w}, & u=0 & \text { at } r=r_{0} \\
T^{\prime}=T_{\infty}^{\prime}, \quad v=0, \varphi=\varphi_{\infty}, \quad u=0 & \text { at } x=0 \\
T^{\prime} \rightarrow T_{\infty}^{\prime}, \quad v \rightarrow 0, \quad \varphi \rightarrow \varphi_{\infty}, \quad u \rightarrow 0, \quad \frac{\partial u}{\partial r} \rightarrow 0 & \text { as } r \rightarrow \infty
\end{array}
$$

The following dimensionless quantities are now introduced:

$$
\begin{aligned}
& X=G r^{-1} \frac{x}{r_{0}}, \quad R=\frac{r}{r_{0}}, \quad U=\frac{G r^{-1} u r_{0}}{\vartheta}, \quad V=\frac{v r_{0}}{\vartheta}, \quad t=\frac{\vartheta t^{\prime}}{r_{0}^{2}}, \\
& T=\frac{T^{\prime}-T_{\infty}^{\prime}}{T_{w}^{\prime}-T_{\infty}^{\prime}}, \quad G r=\frac{\mathrm{g}^{\prime} \beta_{T} r_{0}{ }^{3}\left(T_{w}^{\prime}-T_{\infty}^{\prime}\right)\left(1-\varphi_{\infty}\right) \rho_{f_{\infty}}}{\vartheta^{2} \rho_{f}}, \quad \Theta=\frac{\left(\varphi-\varphi_{\infty}\right)}{\left(\varphi_{w}-\varphi_{\infty}\right)}, \quad \operatorname{Pr}=\frac{\vartheta}{\alpha},
\end{aligned}
$$


$\alpha_{1}=\frac{\alpha_{1}{ }^{*}}{\rho_{f} r_{0}{ }^{2}}, \quad \alpha_{2}=\frac{\alpha_{2}{ }^{*}}{\rho_{f} r_{0}{ }^{2}}, \quad \quad \beta=\frac{\beta_{3}{ }^{*} \vartheta}{\rho_{f} r_{0}{ }^{4}}, \quad L e=\frac{\vartheta}{D_{B}}, \quad \omega=\frac{(\rho C)_{p}}{(\rho C)_{f}}$,

$N r=\frac{\left(\rho_{p}-\rho_{f_{\infty}}\right)\left(\varphi_{w}-\varphi_{\infty}\right)}{\rho_{f_{\infty}} \beta_{T}\left(T_{w}^{\prime}-T_{\infty}^{\prime}\right)\left(1-\varphi_{\infty}\right)}, \quad N b=\frac{(\rho C)_{p} D_{B}\left(\varphi_{w}-\varphi_{\infty}\right)}{(\rho C)_{f} \vartheta}, \quad N t=\frac{(\rho C)_{p} D_{T}\left(T_{w}^{\prime}-T_{\infty}^{\prime}\right)}{(\rho C)_{f} \vartheta T_{\infty}^{\prime}}$

Implementing Eqn. (12) in the conservation Eqns. (6) - (10) and Eqn. (11), hence, the following system of non-dimensional governing equations emerges:

$$
\begin{aligned}
\frac{\partial U}{\partial X}+\frac{\partial V}{\partial R}+\frac{V}{R}= & 0 \\
U \frac{\partial U}{\partial X}+V \frac{\partial U}{\partial R}+ & \frac{\partial U}{\partial t}=T-N r \Theta+\frac{1}{R} \frac{\partial}{\partial R}\left(R \frac{\partial U}{\partial R}\right)+\alpha\left[\frac{\partial^{3} U}{\partial R^{2} \partial t}+\frac{1}{R} \frac{\partial^{2} U}{\partial R \partial t}+U \frac{\partial^{3} U}{\partial X \partial R^{2}}+V \frac{\partial^{3} U}{\partial R^{3}}+\right. \\
& \left.\frac{U}{R} \frac{\partial^{2} U}{\partial X \partial R}+\frac{V}{R} \frac{\partial^{2} U}{\partial R^{2}}+2 \frac{\partial V}{\partial R} \frac{\partial^{2} U}{\partial R^{2}}+3 \frac{\partial^{2} U}{\partial R^{2}} \frac{\partial U}{\partial X}+\frac{\partial U}{\partial R} \frac{\partial^{2} V}{\partial R^{2}}+4 \frac{\partial U}{\partial R} \frac{\partial^{2} U}{\partial X \partial R}+\frac{1}{R} \frac{\partial U}{\partial R} \frac{\partial V}{\partial R}+\frac{3}{R} \frac{\partial U}{\partial X} \frac{\partial U}{\partial R}\right] \\
& +\alpha_{2}\left[\frac{2}{R} \frac{\partial U}{\partial R}\left(\frac{\partial V}{\partial R}+\frac{\partial U}{\partial X}\right)+2 \frac{\partial^{2} U}{\partial R^{2}}\left(\frac{\partial U}{\partial X}+\frac{\partial V}{\partial R}\right)+2 \frac{\partial U}{\partial R}\left(\frac{\partial^{2} V}{\partial R^{2}}+2 \frac{\partial^{2} U}{\partial X \partial R}\right)\right] \\
& +\beta\left[\frac{2(G r)^{2}}{R}\left(\frac{\partial U}{\partial R}\right)^{3}+\left(\frac{\partial U}{\partial R}\right)^{2}\left(6(G r)^{2} \frac{\partial^{2} U}{\partial R^{2}}+4 \frac{\partial^{2} U}{\partial X^{2}}\right)+2 \frac{\partial^{2} U}{\partial X \partial R} \frac{\partial U}{\partial X} \frac{\partial U}{\partial R}\right] \\
U \frac{\partial T}{\partial X}+V \frac{\partial T}{\partial R}+ & \frac{\partial T}{\partial t}=\frac{1}{P r}\left(\frac{\partial^{2} T}{\partial R^{2}}+\frac{1}{R} \frac{\partial T}{\partial R}\right)+N b \frac{\partial \Theta}{\partial R} \frac{\partial T}{\partial R}+N t\left(\frac{\partial T}{\partial R}\right)^{2} \\
U \frac{\partial \Theta}{\partial X}+V \frac{\partial \Theta}{\partial R}+ & \frac{\partial \Theta}{\partial t}=\frac{1}{L e}\left(\frac{\partial^{2} \Theta}{\partial R^{2}}+\frac{1}{R} \frac{\partial \Theta}{\partial R}\right)+\frac{1}{L e} \frac{N t}{N b}\left(\frac{\partial^{2} T}{\partial R^{2}}+\frac{1}{R} \frac{\partial T}{\partial R}\right)
\end{aligned}
$$

$$
\begin{array}{cll}
t \leq 0: T=0, \quad V=0, \quad \Theta=0, \quad U=0 & \text { for all } X \text { and } R \\
t>0: T=1, \quad V=0, \quad \Theta=1, \quad U=0 & \text { at } R=1 \\
T=0, \quad V=0, \quad \Theta=0, \quad U=0 & \text { at } X=0 \\
T \rightarrow 0, \quad V \rightarrow 0, \quad \Theta \rightarrow 0, \quad U \rightarrow 0, \frac{\partial U}{\partial R} \rightarrow 0 & \text { as } R \rightarrow \infty
\end{array}
$$




\section{FINITE DIFFERENCE SOLUTION PROCEDURE}

The non-dimensional model derived in Eqns. (13)-(17) does not yield analytical solutions. Recourse must therefore be made to a numerical scheme which provides an approximate solution with high accuracy. In this regard the implicit finite difference Crank-Nicolson scheme [57] is adopted which is unconditionally stable.

\subsection{Formation of discretized equations}

The discretized finite difference versions of Eqns. (13) - (16) are given in the Appendix section, Eqns. (A.1) - (A.4). The chosen flow domain for the boundary layer regime is $X_{\min }=$ $0, X_{\max }=1, R_{\min }=1$ and $R_{\max }=20$ (where $R_{\max }$ implies to $R=\infty$ ). The discretized Eqns. (A.3) (or (A.4)) and (A.2) at specific ' $f$ - level' and at any mesh point $(f, g)$ constitute a 'tridiagonal' and 'penta-diagonal' system of equations which are respectively represented as:

$A_{1} \delta_{f, g-1}^{h+1}+B_{1} \delta_{f, g}^{h+1}+C_{1} \delta_{f, g+1}^{h+1}=D_{1}$

$A_{2} \gamma_{f, g-2}^{h+1}+B_{2} \gamma_{f, g-1}^{h+1}+C_{2} \gamma_{f, g}^{h+1}+D_{2} \gamma_{f, g+1}^{h+1}+E_{2} \gamma_{f, g+2}^{h+1}=F_{2}$

This system of Eqs. (18) and (19) at $(h+1)^{\text {th }}$ level are solved using 'tridiagonal' [57] and 'pentadiagonal' [58] algorithms, where $\delta$ and $\gamma$ specify $T$ (or $\Theta$ ) and $U$, respectively. Further details on this computational procedure are provided in Reddy et al. [42], [43]. Initialization of the iterative scheme starts with the computation of volume fraction (nano-particle species concentration function, $\Theta$ ) by solving discretized volumetric Eqn., followed by computation of the temperature, $T$, which is solved via thermal Eqn.. Thereafter, the solution procedure is carried out to determine the velocity components by solving the momentum and mass conservative equations. 


\subsection{Validation of the Crank-Nicolson numerical scheme}

Validation of the Crank-Nicolson numerical solutions is now described. This is achieved with a two-tier approach. Firstly, mesh independence must be confirmed. Secondly benchmarking with previously published results is also conducted for the special case of Newtonian thermal convection nanofluid flow from a vertical cylinder against the results of Chamkha et al. [23] and also the purely Newtonian case of Rani and Kim [59] (neglecting nano-particle effects).

\section{Mesh Independence}

For the current study, an economically consistent grid-system has been proposed by conducting the 'grid-independency test' for chosen grid-sizes. Table 1 provides the suitable grid-size, i.e., 100 X 500 which successfully achieves accurate results (further mesh refinement does not yield any significant improvement in accuracy). Similarly, 'time-independency test' provides the optimal specific time-step size $\Delta t(t=h \Delta t, h=0,1,2, \ldots)=0.01$ as elucidated in Table 2 . For both tests nano effects are fixed i.e., $N b=N t=N r=1.0, L e=10.0$.

\section{Benchmark with earlier special cases}

The radial flow profiles of $U, T$, and $\Theta$ are plotted graphically. A detailed comparative study of fluid flow with previous results is conducted to ensure the exactness of the solution of current flow-model.

- Firstly only Newtonian fluid flow (i.e., $\alpha_{1}=\alpha_{2}=\beta=0, N b=N t=0$ with the nanoparticle species Eqn. (16) is neglected) is considered by ignoring the nanofluid and third grade viscoelastic parameters in the present model and matching with the results of 
Rani and Kim [59]. The comparison (refer to Fig. 2) confirms the high accuracy of the Crank-Nicolson finite difference numerical code.

- Secondly a Newtonian nanofluid is considered wherein nanoparticle effects are studied whereas viscoelastic parameters are negated (i.e., $\alpha_{1}=\alpha_{2}=\beta=0$, and the nanoparticle species Eqn. (16) is included) and in this case, we verify the solutions with those in Chamkha et al. [23]. Again, very close correlation is achieved, as depicted in Fig. 3.

The above comparative studies provide high levels of confidence in the accuracy of the adopted numerical method (Crank-Nicolson scheme).

\section{Table1. Grid independent test:}

\begin{tabular}{|c|c|c|c|}
\hline Grid size & $\begin{array}{c}\overline{\boldsymbol{C}}_{\boldsymbol{f}} \text { values for } \boldsymbol{P r}=0.7, \boldsymbol{G r} \\
=10.0, \\
\boldsymbol{\alpha}_{\mathbf{1}}=\boldsymbol{\alpha}_{\mathbf{2}}=\boldsymbol{\beta}=\mathbf{0 . 2}\end{array}$ & $\begin{array}{c}\overline{\boldsymbol{N u}} \text { values for } \boldsymbol{P r}=0.7, \\
\boldsymbol{G r}=10.0, \\
\boldsymbol{\alpha}_{1}=\boldsymbol{\alpha}_{2}=\boldsymbol{\beta}=\mathbf{0 . 2}\end{array}$ & $\begin{array}{c}\boldsymbol{S h} \text { values for } \boldsymbol{P r}= \\
0.7, \boldsymbol{G r}=10.0 \\
\boldsymbol{\alpha}_{\mathbf{1}}=\boldsymbol{\alpha}_{2}=\boldsymbol{\beta} \\
=\mathbf{0 . 2}\end{array}$ \\
\hline $25 \times 125$ & 0.15313330 & 0.33480950 & 1.64730800 \\
\hline $50 \times 250$ & 0.15350400 & 0.33116020 & 1.69171300 \\
\hline $100 \times 500$ & 0.15362190 & 0.32950480 & 1.71289300 \\
\hline $200 \times 1000$ & 0.15376790 & 0.32887780 & 1.71927600 \\
\hline
\end{tabular}


Table2. Time-independence test:

\begin{tabular}{|c|c|c|c|}
\hline $\begin{array}{l}\text { Time step } \\
\text { size }(\Delta t)\end{array}$ & $\begin{array}{c}\overline{\boldsymbol{C}}_{\boldsymbol{f}} \text { values for } \boldsymbol{P r}=0.7, \\
\boldsymbol{G r}=10.0, \\
\boldsymbol{\alpha}_{\mathbf{1}}=\boldsymbol{\alpha}_{\mathbf{2}}=\boldsymbol{\beta}=\mathbf{0 . 2}\end{array}$ & $\begin{array}{c}\overline{\boldsymbol{N u}} \text { values for } \boldsymbol{P r}=0.7 \\
\boldsymbol{G r}=10.0 \\
\boldsymbol{\alpha}_{\mathbf{1}}=\boldsymbol{\alpha}_{2}=\boldsymbol{\beta}=\mathbf{0 . 2}\end{array}$ & $\begin{array}{c}\boldsymbol{S h} \text { values for } \boldsymbol{P r}= \\
0.7, \boldsymbol{G r}=10.0 \\
\boldsymbol{\alpha}_{1}=\boldsymbol{\alpha}_{2}=\boldsymbol{\beta} \\
=\mathbf{0 . 2}\end{array}$ \\
\hline 0.1 & 0.15362970 & 0.32961000 & 1.71302400 \\
\hline 0.08 & 0.15363250 & 0.32961430 & 1.71304800 \\
\hline 0.05 & 0.15362810 & 0.32958360 & 1.71298500 \\
\hline 0.03 & 0.15362590 & 0.32956800 & 1.71295300 \\
\hline 0.01 & 0.15362190 & 0.32950480 & 1.71289300 \\
\hline
\end{tabular}

\section{GRAPHICAL RESULTS AND DISCUSSION}

The steady-state behaviour of velocity, temperature and volume fraction of the thirdgrade nanofluid are studied considering thermophoresis and Brownian effects. Also, the impact of third-grade fluid parameter at constant Grashof number on the flow characteristics under both transient and steady-state conditions are visualized graphically. Transient results of skinfriction, heat transfer rate and Sherwood number for variation of a third-grade parameter are additionally presented. In this respect, the following subsections provide a comprehensive discussion.

\section{Flow profiles for variation of third-grade parameter $(\beta)$}

At a location near to the hot cylindrical wall, steady and unsteady flow profiles are computed for the effect of different values of a third-grade parameter $(\beta)$. Figs. 4, 5 and 6 display the 
effect of $\beta$ on velocity, temperature and volume fraction at different times. From Fig. 4, it is observed that the transient velocity of the fluid at $(1,1.912)$ decreases as $\beta$ increases. Figures $5 \& 6$ show that temperature and volume fraction of the fluid at $(1,1.152)$ increase for amplifying values of $\beta$. It is known that third-grade fluid parameter describes the viscoelastic effect in polymers (i.e. the relative contribution of viscous and elastic forces) $[35,36]$. As it increases, the viscosity dominates and the elastic nature of the fluid recedes which manifests in a deceleration in the nano-polymer flow. The decrease in the velocity of the nano-polymer fluid is clearly captured in Fig. 4. Also, due to high viscous and lower elastic effects, there will be enhanced collisions of the fluid particles (elevated momentum diffusion) which will result in heating of the nano-polymer. Temperatures are therefore elevated with greater third-grade material parameter values (refer Fig. 5). Similarly, this heating of the boundary layer (associated with enhanced thermal diffusion) will simultaneously encourage nano-particle diffusion which leads to an elevation in nanoparticle volume fraction magnitudes (Fig. 6). It is noticeable in each profile that $U, T$, and $\Theta$ - curves are overlapped. Initially heat conduction dominates over the convection effect and after particular time intervals, the curves deviate from each other showing the buoyancy-induced convection flow takes over i.e. thermal convection current swamp the influence of thermal conduction.

Figures 7, $8 \& 9$ depict the steady-state velocity, temperature and volume fraction profiles at the location, $X=1.0$. Figure 7 shows steady-state velocity curves for variations of $\beta$. The shape of all curves for different $\beta$ values is similar and is characterized by a particular peak value (refer Fig. 7). However, the location of this peak is displaced further from the cylinder surface with increasing third-grade material parameter values. In the flow-region $0<R<$ 4.8, the velocity $(U)$ profile shows a decreasing trend and this is subsequently reversed further from the cylinder surface. A decrement in the fluid velocity is computed which is similar to the response in the transient condition. From Fig. 8, it is noticeable that, temperature curves start 
from boundary condition $T=1$ and run radially downwards to $T=0$, i.e., free stream temperature. Since, amplifying $\beta$ values promotes the viscoelastic behavior of the third-grade nanofluid, this exacerbates collisions of fluid molecules and increases the temperature value and also thickens the thermal boundary layer. Similarly, nanoparticle volume fraction curves also follow the same trend as temperature profile and a thickening of the nanoparticle volume fraction (species) boundary layer is also induced with increasing $\beta$ (refer Fig. 9).

\section{Effect of Brownian motion parameter}

The random motion of the nanoparticles within the base fluid, i.e., 'third-grade fluid' is characterized as Brownian motion. It can be considered as a measure of the concentration gradient of nanoparticles. Here Figs $10,11 \& 12$ illustrate the effect of Brownian motion parameter on non-dimensional velocity, temperature and volume fraction, respectively. As $\mathrm{Nb}$ (Brownian motion parameter) increases the disordered motion of nanoparticle increases via ballistic collisions and therefore particles move with greater velocity. It is evident from Fig. 10 that steady-state velocity is an increasing function of $\mathrm{Nb}$. The pattern of velocity curves is the same irrespective of the value of $N b$ i.e. profiles ascend from the no-slip wall value, $U=0$, accelerate to $U_{\max }$ then drop to $U=0$ at steady-state. The magnitude of $U_{\max }$ is enhanced with elevation in $N b$. Also, the intensification in disordered motion corresponding to larger $\mathrm{Nb}$ values increases the collisions of nanoparticles. This results in heating of the nano-polymer regime which manifests in elevation in temperature and significant thickening of the thermal boundary layer with greater $\mathrm{Nb}$ (Fig. 11). Brownian motion clearly encouraged thermal diffusion in viscoelastic nanofluids. All temperature $(T-)$ curves initialize with $T=1$ (maximum temperature at steady condition) then falls drastically to $T=0$. Figure 12 shows that nano-particle volume fraction (concentration) i.e. $\Theta$ - curves also follow similar pathways to the temperature curves, although $\Theta$ magnitudes are markedly less than temperature 
magnitudes. The randomness of nanoparticles therefore also impacts on the volume fraction of nanoparticle within third-grade fluid i.e., as $\mathrm{Nb}$ rises volume fraction decreases. This is also consistent with the original physics underlying the Buongiorno model since larger $\mathrm{Nb}$ values correspond to larger sized nano-particles indicating a reduction in volume fraction.

\section{Effect of thermophoresis parameter}

The temperature gradient produced due to density difference is more prominent in natural convective flows. This temperature gradient enhances the thermophoresis forces and certainly impacts on the free-convective flow. Thermophoretic forces drive the migration of nanoparticle across the nanofluid. Thermophoresis is a force generated by the temperature gradient between the heated nanofluid and cooler boundary and this mobilizes transport of nano-particles towards the colder boundary. The velocity profile evidently exhibits an increasing trend with greater values of thermophoresis parameter $(N t)$ (see Fig. 13). The magnitude of maximum velocity increases for rising values of $N t$. Greater $N t$ values promote the fast movement of nanoparticles, which in turn, increases the temperature, i.e., the thermal boundary layer thickens at a steady-state condition which is reflected in Fig. 14. Similarly, this thermophoretic forces also influences volume fraction of the nanoparticles. Referring to Fig. 15 it is apparent that higher $N t$ is associated with a lower volume fraction of nanoparticle and this observation is reversed after $R=2.8$. From all graphs for variation of $N t$, it is observed that the change in flow variables remain almost invariable after $N t=3$.

\section{Effect of Lewis number}

In thermo-solutal transport phenomena problems, Lewis number signifies the relation between thermal and mass diffusivities i.e. the rates at which heat and species (nano-particles) diffuse in the base fluid (polymer). From Fig. 16, it is to be noted that as Lewis number increases, the flow is accelerated i.e. the maximum velocity attains a higher value. However, the converse 
effect is induced in the temperature and volume fraction profiles. Both temperature and nanoparticle volume fraction are depressed with greater Lewis number. There is therefore a reduction in both thermal and nano-particle species (concentration) boundary layer thicknesses with higher values of Le (Lewis number). It is also noteworthy that nano-particle volume fraction $(\Theta)$ profiles (Fig. 18) are consistently lower than temperature $(T)$ profiles (Fig. 17). However the effect of $L e$ is more prominent on nano-particle volume fraction than on temperature.

\section{Effect of Buoyancy ration parameter}

Figures 19, $20 \& 21$ illustrate the evolution in non-dimensional flow variables (velocity, temperature and volume fraction respectively) with variation buoyancy ratio parameter $(\mathrm{Nr})$. The impact of $N r$ on flow variables is weaker than that of the other parameters examined earlier (i.e. $N b, N t$ and $\beta$ ). Figure 19 depicts the influence of $N r$ on velocity profile at the steady-state condition. It is noticeable that as $\mathrm{Nr}$ increases, the velocity profile exhibits a decreasing trend. In other words, with greater thermal buoyancy force relative to nano-particle species buoyancy force (both forces are equivalent when $N r=1$ ) the boundary layer flow is decelerated, and momentum boundary layer thickness is increased. Increasing $N r$ however induces a very weak elevation in temperature, $T$ (see Fig. 20) and nano-particle volume fraction, $\Theta$ (see Fig. 21) profiles, i.e., as $N r$ values upsurge both thermal and nano-particle concentration boundary layer thicknesses are weakly increased. Generally lower magnitudes of volume fraction profile are computed relative to temperature over the same increment in buoyancy ratio parameter.

\section{Coefficient of skin-friction, heat transfer rate and nano-particle Sherwood number}

In coating dynamics and materials processing, skin friction coefficient $\left(\overline{C_{f}}\right)$ (Fig. 22), wall heat transfer rate $(\overline{N u})$ (Fig. 23) and nano-particle wall mass transfer rate i.e. Sherwood number (Sh) (Fig. 24) are important design quantities. Here, their non-dimensional forms are given by: 
$\overline{C_{f}}=\int_{0}^{1}\left(\frac{\partial U}{\partial R}\right)_{R=1} d X, \overline{N u}=-\int_{0}^{1}\left(\frac{\partial T}{\partial R}\right)_{R=1} d X \quad \& \quad S h=-\int_{0}^{1}\left(\frac{\partial \Theta}{\partial R}\right)_{R=1} d X$

The effect of third-grade fluid parameter $\beta$ on skin-friction is depicted in Fig. 22. It is observed that as $\beta$ is raised, $\overline{C_{f}}$ values decrease i.e. the flow is decelerated which concurs with the earlier velocity distribution results in Fig. 4. As viscoelastic nature $(\beta)$ of the nanofluid increases, the boundary layer flow is impeded which decreases fluid velocity adjacent to the heated cylinder surface (wall). Figure 23 depicts the $\overline{N u}$ profiles at $R=1$ (hot wall). At $t=0$ (starting time), all $\overline{N u}$ curves follow the same pattern i.e. they descend from a peak and thereafter ascend to reach the steady-state with subsequent time elapse. $\overline{N u}$ is decreasing function for rising values of $\beta$. Higher $\beta$ values signify more nano-particle collisions due to the viscoelastic behavior of third-grade nanofluid and this elevates the temperature of the nanofluid regime which is evident from Fig. 5. The heating of the boundary layer implies a decrease in the rate of heat transfer to the cylinder surface i.e. a reduction in $\overline{N u}$ (see Fig. 23). Fig. 24 shows the evolution in Sherwood number with variation of $\beta$ at $R=1$. Since greater third-grade viscoelastic material parameter values are associated with an enhancement in nano-particle volume fraction magnitudes (Fig. 6), the rate of nano-particle species transfer to the wall is reduced. Sherwood number magnitudes are therefore decreased with greater values of $\beta$. Again all Sherwood number profiles descend monotonically until the steady-state magnitudes are attained.

\section{Isotherms and Stream function}

Isotherms are drawn in a two-dimensional rectangular plane (i.e., $0<X<1,0<R<6$ ) for different values of the third-grade fluid parameter, $\beta$. Isotherms signify the temperature values in the whole flow-domain (two-dimensional plane) within the thermal boundary layer. From Fig. 25, it is evident that as third-grade fluid parameter values amplify isotherms are slightly 
displaced away from the heated cylinder surface. A dimensionless stream function $(\psi)$ is used to express the hydrodynamics of the nanofluid and must satisfy the equation of mass conservation (refer to Eq. 13). The stream function can be defined with velocity vectors in the two-dimensional plane as:

$U=\left(\frac{1}{R} \frac{\partial \psi}{\partial R}\right) \quad$ and $\quad V=-\left(\frac{1}{R} \frac{\partial \psi}{\partial X}\right)$

Equation (21) yields:

$\frac{\partial^{2} \psi}{\partial R^{2}}+\frac{\partial^{2} \psi}{\partial X^{2}}=R\left(\frac{\partial U}{\partial R}-\frac{\partial V}{\partial X}\right)+U$

A second-order central difference formula is adopted to calculate $\psi$. Streamlines are drawn in a two-dimensional rectangular plane, i.e., $0<X<1,0<R<6$ for the variation of thirdgrade fluid parameter in Fig. 26. Here, the deviation of streamlines away from the heated surface is clearly observed with increasing values of the third-grade material parameter, $\beta$.

\section{Contour graphs}

The contour lines are drawn in a two-dimensional rectangular coordinate plane, i.e., $0<X<$ 1, $0<R<6$ for all flow variables $(U, T \& \Theta)$ of third-grade nanofluid (Fig. 27 (a)) and Newtonian nanofluid (Fig. 27 (b)). These contour lines drawn for third-grade nanofluid are moving away from the hot cylindrical wall noticeably as compared to the Newtonian nanofluid, i.e., shifting of velocity, temperature and nano-particle volume fraction contours away from the heated cylinder surface for the third-grade nanofluid is observed. These observations confirm that non-Newtonian nanofluids (i.e. third-grade viscoelastic characteristics) induce a measurable deviation in thermo-solutal characteristics relative to Newtonian nanofluids.

\section{CONCLUSIONS}


Motivated by nano-polymeric thermal coating dynamics, in the current article a mathematical model has been presented for unsteady free-convective laminar flow of third-grade viscoelastic nanofluid from a semi-infinite vertical isothermal cylinder. Non-Newtonian behavior has been modelled with the thermodynamically robust third-grade Reiner-Rivlin model which accurately simulates polymer rheology. Nanoscale effects has been studied with the Buongiorno two-component nanofluid model which features Brownian motion and thermophoresis effects. The non-dimensional governing highly coupled, nonlinear, multidegree partial differential equations for mass, momentum, energy and nano-particle species (volume fraction) conservation have been solved with an implicit Crank-Nicolson numerical scheme subject to appropriate initial and boundary conditions. Validation of the numerical solutions with earlier published results on Newtonian and Newtonian nanofluids has been included. Mesh and time-independence numerical grid studies have also been conducted. The present computations have shown that:

- With increasing third-grade viscoelastic material parameter or buoyancy ratio parameter, the velocity decreases whereas temperature and nano-particle volume fraction increase.

- For higher values of Brownian motion parameter, steady-state velocity and temperature increase whereas steady-state nano-particle volume fraction decreases.

- With greater values of thermophoresis parameter, thicker velocity (or temperature) and thinner nano-particle species boundary layers are observed.

- With increasing Lewis number, non-dimensional steady-state temperature and nanoparticle volume fraction are decreased whereas velocity is enhanced.

- For rising values of the third-grade parameter, wall rate of heat transfer (Nusselt number), skin friction and wall nano-particle mass transfer rate (Sherwood number) are diminished at the heated cylindrical surface (wall). 
- A displacement in velocity, temperature and nano-particle volume fraction contours is observed away from the heated cylinder surface for the third-grade nanofluid relative to the Newtonian case.

- Generally, the results obtained for third-grade viscoelastic nanofluids vary considerably from those obtained for Newtonian nanofluids.

The current study has neglected viscous dissipation effects and has been confined to Boussinesq flow. Future investigations will consider dissipative non-Boussinesq [60] coating flows of non-Newtonian nanofluids and will be communicated imminently.

\section{ACKNOWLEDGEMENTS}

The authors appreciate greatly the comments of the reviewers which have served to improve the present work.

\section{APPENDIX}

The discretized finite difference equations for the Eqs. (13) - (16) are as follows:

$\frac{U_{f, g}^{h+1}-U_{f-1, g}^{h+1}+U_{f, g}^{h}-U_{f-1, g}^{h}}{2 \Delta X}+\frac{V_{f, g}^{h+1}-V_{f, g-1}^{h+1}+V_{f, g}^{h}-V_{f, g-1}^{h}}{2 \Delta R}+V_{f, g}^{h+1}(J R)=0$

$$
\begin{aligned}
& \frac{U_{f, g}^{h+1}-U_{f, g}^{h}}{\Delta t}+U_{f, g}^{h} \frac{\left(U_{f, g}^{h+1}-U_{f-1, g}^{h+1}+U_{f, g}^{h}-U_{f-1, g}^{h}\right)}{2 \Delta X}+V_{f, g}^{h} \frac{\left(U_{f, g+1}^{h+1}-U_{f, g-1}^{h+1}+U_{f, g+1}^{h}-U_{f, g-1}^{h}\right)}{4 \Delta R} \\
& =\frac{T_{f, g}^{h}+T_{f, g}^{h+1}}{2}-N r \frac{\Theta_{f, g}^{h}+\Theta_{f, g}^{h+1}}{2}+J R \frac{\left(U_{f, g+1}^{h+1}-U_{f, g-1}^{h+1}+U_{f, g+1}^{h}-U_{f, g-1}^{h}\right)}{4 \Delta R}
\end{aligned}
$$




$$
\begin{aligned}
& +\frac{\left(U_{f, g-1}^{h+1}-2 U_{f, g}^{h+1}+U_{f, g+1}^{h+1}+U_{f, g-1}^{h}-2 U_{f, g}^{h}+U_{f, g+1}^{h}\right)}{2(\Delta R)^{2}}+\alpha_{1}\left[\frac{\left(U_{f, g-2}^{h+1}-2 U_{f, g}^{h+1}+U_{f, g+2}^{h+1}-U_{f, g-2}^{h}+2 U_{f, g}^{h}-U_{f, g+2}^{h}\right)}{4(\Delta R)^{3}(\Delta t)}\right. \\
& +\frac{\left(U_{f, g+1}^{h+1}-U_{f, g-1}^{h+1}+U_{f, g+1}^{h}-U_{f, g-1}^{h}\right)}{2(\Delta R)(\Delta t)}+V_{f, g}^{h} \frac{\left(U_{f, g}^{h+1}-2 U_{f, g+1}^{h+1}+2 U_{f, g-1}^{h+1}-U_{f, g-2}^{h+1}+U_{f, g+2}^{h}-2 U_{f, g+1}^{h}+2 U_{f, g-1}^{h}-U_{f, g-2}^{h}\right)}{4(\Delta R)^{3}} \\
& +\frac{\left(V_{f, g+1}^{h}-V_{f, g-1}^{h}\right)}{2 \Delta R} \frac{\left(U_{f, g-1}^{h+1}-2 U_{f, g}^{h+1}+U_{f, g+1}^{h+1}+U_{f, g-1}^{h}-2 U_{f, g}^{h}+U_{f, g+1}^{h}\right)}{(\Delta R)^{2}} \\
& +3 \frac{\left(U_{f, g}^{h}-U_{f-1, g}^{h}\right)}{(\Delta X)} \frac{\left(U_{f, g-1}^{h+1}-2 U_{f, g}^{h+1}+U_{f, g+1}^{h+1}+U_{f, g-1}^{h}-2 U_{f, g}^{h}+U_{f, g+1}^{h}\right)}{2(\Delta R)^{2}}+\frac{\left(V_{f, g-1}^{h}-2 V_{f, g}^{h}+V_{f, g+1}^{h}\right)}{4(\Delta R)^{2}}
\end{aligned}
$$$$
\frac{\left(U_{f, g+1}^{h+1}-U_{f, g-1}^{h+1}+U_{f, g+1}^{h}-U_{f, g-1}^{h}\right)}{(\Delta R)}+\frac{\left(U_{f, g+1}^{h}-U_{f, g-1}^{h}\right)}{\Delta R} \frac{\left(U_{f, g+1}^{h+1}-U_{f-1, g-1}^{h+1}+U_{f, g+1}^{h}-U_{f-1, g-1}^{h}\right)}{2(\Delta X)(\Delta R)}+
$$$$
(J R) V_{f, g}^{h} \frac{\left(U_{f, g-1}^{h+1}-2 U_{f, g}^{h+1}+U_{f, g+1}^{h+1}+U_{f, g-1}^{h}-2 U_{f, g}^{h}+U_{f, g+1}^{h}\right)}{2(\Delta R)^{2}}+
$$$$
(J R) U_{f, g}^{h} \frac{\left(U_{f, g+1}^{h+1}-U_{f-1, g+1}^{h+1}-U_{f, g-1}^{h+1}+U_{f-1, g-1}^{h+1}+U_{f, g+1}^{h}-U_{f-1, g+1}^{h}-U_{f, g-1}^{h}+U_{f-1, g-1}^{h}\right)}{4(\Delta R)(\Delta X)}+
$$$$
\left.3(J R) \frac{\left(U_{f, g}^{h}-U_{f-1, g}^{h}\right)}{(\Delta X)} \frac{\left(U_{f, g+1}^{h+1}-U_{f, g-1}^{h+1}+U_{f, g+1}^{h}-U_{f, g-1}^{h}\right)}{4(\Delta R)}+\frac{3(J R)}{8} \frac{\left(V_{f, g+1}^{h}-V_{f, g-1}^{h}\right)}{\Delta R} \frac{\left(U_{f, g+1}^{h+1}-U_{f, g-1}^{h+1}+U_{f, g+1}^{h}-U_{f, g-1}^{h}\right)}{(\Delta R)^{2}}\right]+
$$$$
\alpha_{2}\left[(J R) \frac{\left(V_{f, g+1}^{h}-V_{f, g-1}^{h}\right)}{\Delta R} \frac{\left(U_{f, g+1}^{h+1}-U_{f, g-1}^{h+1}+U_{f, g+1}^{h}-U_{f, g-1}^{h}\right)}{\Delta R}+(J R) \frac{\left(U_{f, g+1}^{h+1}-U_{f, g-1}^{h+1}+U_{f, g+1}^{h}-U_{f, g-1}^{h}\right)}{2(\Delta R)}\right.
$$$$
\frac{\left(U_{f, g}^{h}-U_{f-1, g}^{h}\right)}{(\Delta X)}+\frac{\left(U_{f, g}^{h}-U_{f-1, g}^{h}\right)}{(\Delta X)} \frac{\left(U_{f, g-1}^{h+1}-2 U_{f, g}^{h+1}+U_{f, g+1}^{h+1}+U_{f, g-1}^{h}-2 U_{f, g}^{h}+U_{f, g+1}^{h}\right)}{(\Delta R)^{2}}+\frac{\left(U_{f, g+1}^{h+1}-U_{f, g-1}^{h+1}+U_{f, g+1}^{h}-U_{f, g-1}^{h}\right)}{2 \Delta R}
$$$$
\frac{\left(V_{f, g-1}^{h}-2 V_{f, g}^{h}+V_{f, g+1}^{h}\right)}{(\Delta R)^{2}}+\frac{\left(U_{f, g+1}^{h}-U_{f, g-1}^{h}\right)}{2(\Delta R)} \frac{\left(U_{f, g+1}^{h+1}-U_{f-1, g-1}^{h+1}+U_{f, g+1}^{h}-U_{f-1, g-1}^{h}\right)}{(\Delta X)(\Delta R)}+\frac{\left(V_{f, g+1}^{h}-V_{f, g-1}^{h}\right)}{2(\Delta R)}
$$$$
\left.\frac{\left(U_{f, g-1}^{h+1}-2 U_{f, g}^{h+1}+U_{f, g+1}^{h+1}+U_{f, g-1}^{h}-2 U_{f, g}^{h}+U_{f, g+1}^{h}\right)}{(\Delta R)^{2}}\right]+\beta\left[(J R)(G r)^{2} \frac{\left(U_{f, g+1}^{h}-U_{f, g-1}^{h}\right)^{3}}{4(\Delta R)^{3}}+\right.
$$$$
3(G r)^{2} \frac{\left(U_{f, g+1}^{h}-U_{f, g-1}^{h}\right)^{2}}{4(\Delta R)^{2}} \frac{\left(U_{f, g-1}^{h+1}-2 U_{f, g}^{h+1}+U_{f, g+1}^{h+1}+U_{f, g-1}^{h}-2 U_{f, g}^{h}+U_{f, g+1}^{h}\right)}{(\Delta R)^{2}}+
$$$$
\frac{\left(U_{f, g+1}^{h}-U_{f, g-1}^{h}\right)^{2}}{(\Delta R)^{2}} \frac{\left(U_{f-1, g}^{h}-2 U_{f, g}^{h}+U_{f+1, g}^{h}\right)}{(\Delta X)^{2}}+\frac{1}{8} \frac{\left(U_{f, g}^{h}-U_{f-1, g}^{h}\right)}{(\Delta X)} \frac{\left(U_{f, g+1}^{h}-U_{f, g-1}^{h}\right)}{(\Delta R)}
$$

$$
\left.\frac{\left(U_{f, g+1}^{h+1}-U_{f-1, g+1}^{h+1}-U_{f, g-1}^{h+1}+U_{f-1, g-1}^{h+1}+U_{f, g+1}^{h}-U_{f-1, g+1}^{h}-U_{f, g-1}^{h}+U_{f-1, g-1}^{h}\right)}{(\Delta X)(\Delta R)}\right]
$$




$$
\begin{aligned}
& \frac{T_{f, g}^{h+1}-T_{f, g}^{h}}{\Delta t}+U_{f, g}^{h} \frac{\left(T_{f, g}^{h+1}-T_{f-1, g}^{h+1}+T_{f, g}^{h}-T_{f-1, g}^{h}\right)}{2 \Delta X}+V_{f, g}^{h} \frac{\left(T_{f, g+1}^{h+1}-T_{f, g-1}^{h+1}+T_{f, g+1}^{h}-T_{f, g-1}^{h}\right)}{4 \Delta R} \\
& =\frac{1}{2 P r} \frac{\left(T_{f, g-1}^{h+1}-2 T_{f, g}^{h+1}+T_{f, g+1}^{h+1}+T_{f, g-1}^{h}-2 T_{f, g}^{h}+T_{f, g+1}^{h}\right)}{(\Delta R)^{2}}+\frac{J R}{4 P r} \frac{\left(T_{f, g+1}^{h+1}-T_{f, g-1}^{h+1}+T_{f, g+1}^{h}-T_{f, g-1}^{h}\right)}{(\Delta R)}+\frac{N b}{8} \frac{\left(\Theta_{f, g}^{h} h+\Theta_{f, g-1}^{h}\right)}{(\Delta R)} \\
& \frac{\left(T_{f, g+1}^{h+1}-T_{f, g-1}^{h+1}+T_{f, g+1}^{h}-T_{f, g-1}^{h}\right)}{(\Delta R)}+\frac{N t}{8} \frac{\left(T_{f, g+1}^{h}-T_{f, g-1}^{h}\right)}{(\Delta R)} \frac{\left(T_{f, g+1}^{h+1}-T_{f, g-1}^{h+1}+T_{f, g+1}^{h}-T_{f, g-1}^{h}\right)}{(\Delta R)} \\
& \frac{\Theta_{f, g}^{h+1}-\Theta_{f, g}^{h}}{\Delta t}+U_{f, g}^{h} \frac{\left(\Theta_{f, g}^{h+1}-\Theta_{f-1, g}^{h+1}+\Theta_{f, g}^{h}-\Theta_{f-1, g}^{h}\right)}{2 \Delta X}+V_{f, g}^{h} \frac{\left(\Theta_{f, g+1}^{h+1}-\Theta_{f, g-1}^{h+1}+\Theta_{f, g+1}^{h}-\Theta_{f, g-1}^{h}\right)}{4 \Delta R} \\
& =\frac{1}{(L e)} \frac{\left(\Theta_{f, g-1}^{h+1}-2 \Theta_{f, g}^{h+1}+\Theta_{f, g+1}^{h+1}+\Theta_{f, g-1}^{h}-2 \Theta_{f, g}^{h}+\Theta_{f, g+1}^{h}\right)}{2(\Delta R)^{2}}+\frac{J R}{(L e)} \frac{\left(\Theta_{f, g+1}^{h+1}-\Theta_{f, g-1}^{h+1}+\Theta_{f, g+1}^{h}-\Theta_{f, g-1}^{h}\right)}{4(\Delta R)} \\
& +\frac{(N t)}{(L e)(N b)} \frac{\left(T_{f, g-1}^{h+1}-2 T_{f, g}^{h+1}+T_{f, g+1}^{h+1}+T_{f, g-1}^{h}-2 T_{f, g}^{h}+T_{f, g+1}^{h}\right)}{2(\Delta R)^{2}}+\frac{(J R)(N t)}{4(L e)(N b)} \frac{\left(T_{f, g+1}^{h+1}-T_{f, g-1}^{h+1}+T_{f, g+1}^{h}-T_{f, g-1}^{h}\right)}{(\Delta R)}
\end{aligned}
$$

\section{FIGURES}

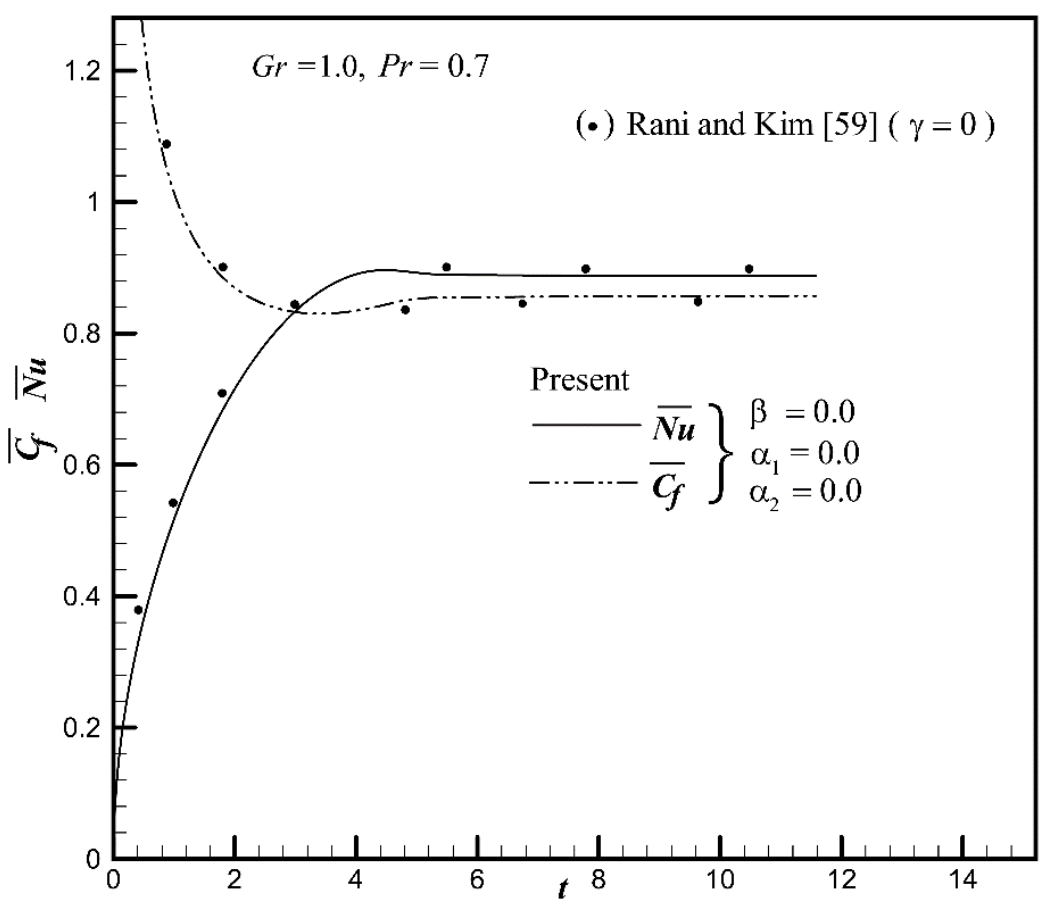

Fig. 2. Comparison study for Newtonian fluid. 


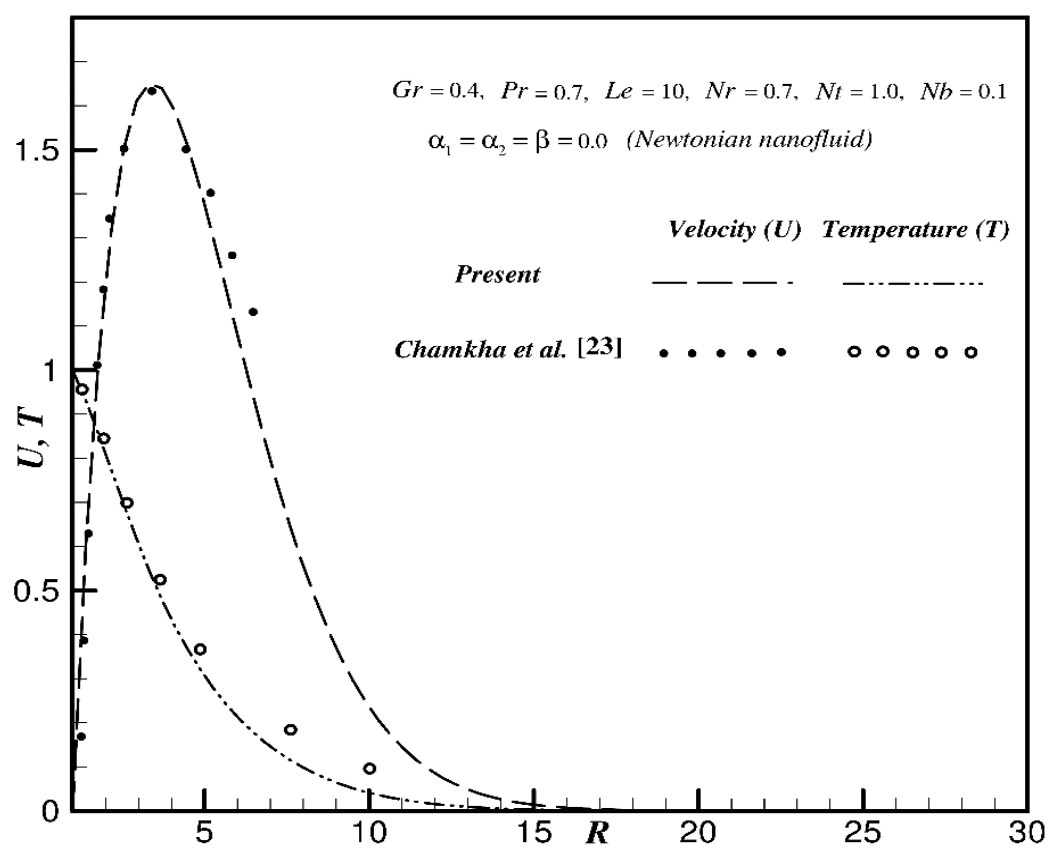

Fig. 3. Comparison study for Newtonian nanofluid.

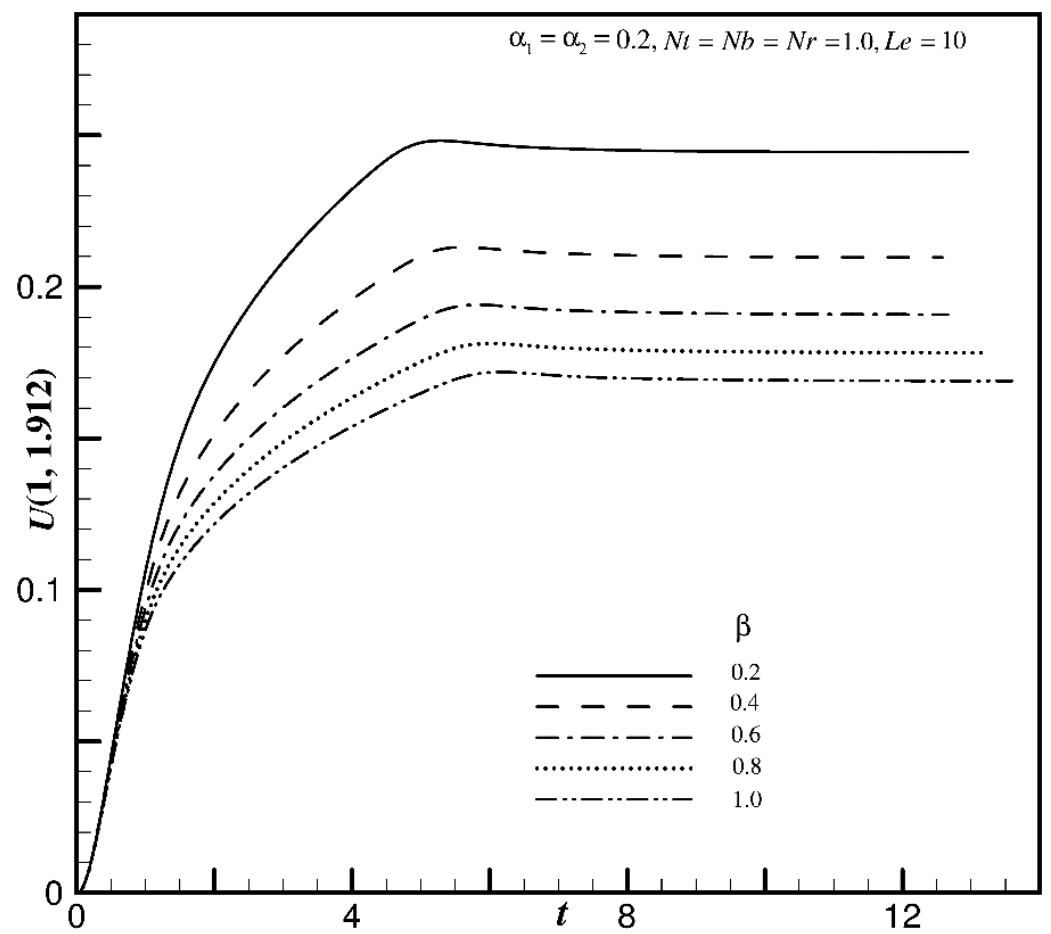

Fig. 4. Unsteady non-dimensional velocity profile against time $(t)$ for variation of third-grade fluid parameter. 


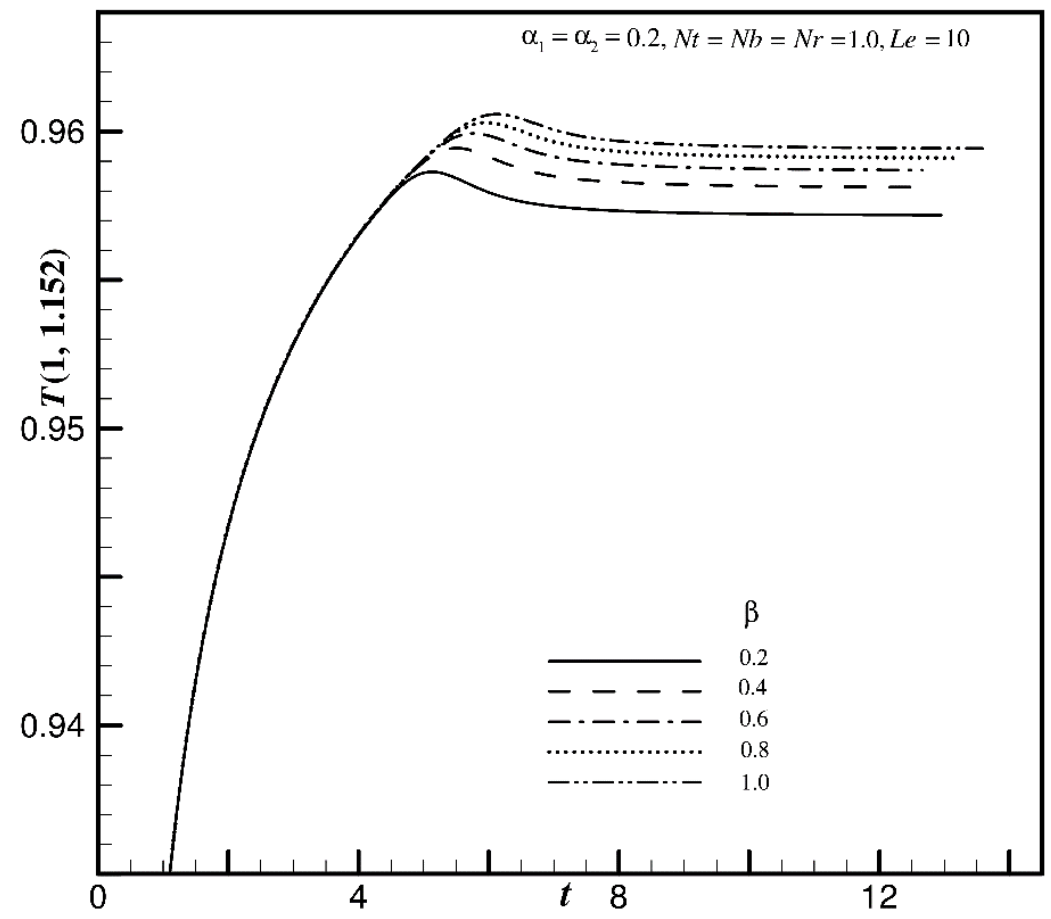

Fig. 5. Unsteady non-dimensional temperature profile against time $(t)$ for variation of thirdgrade fluid parameter.

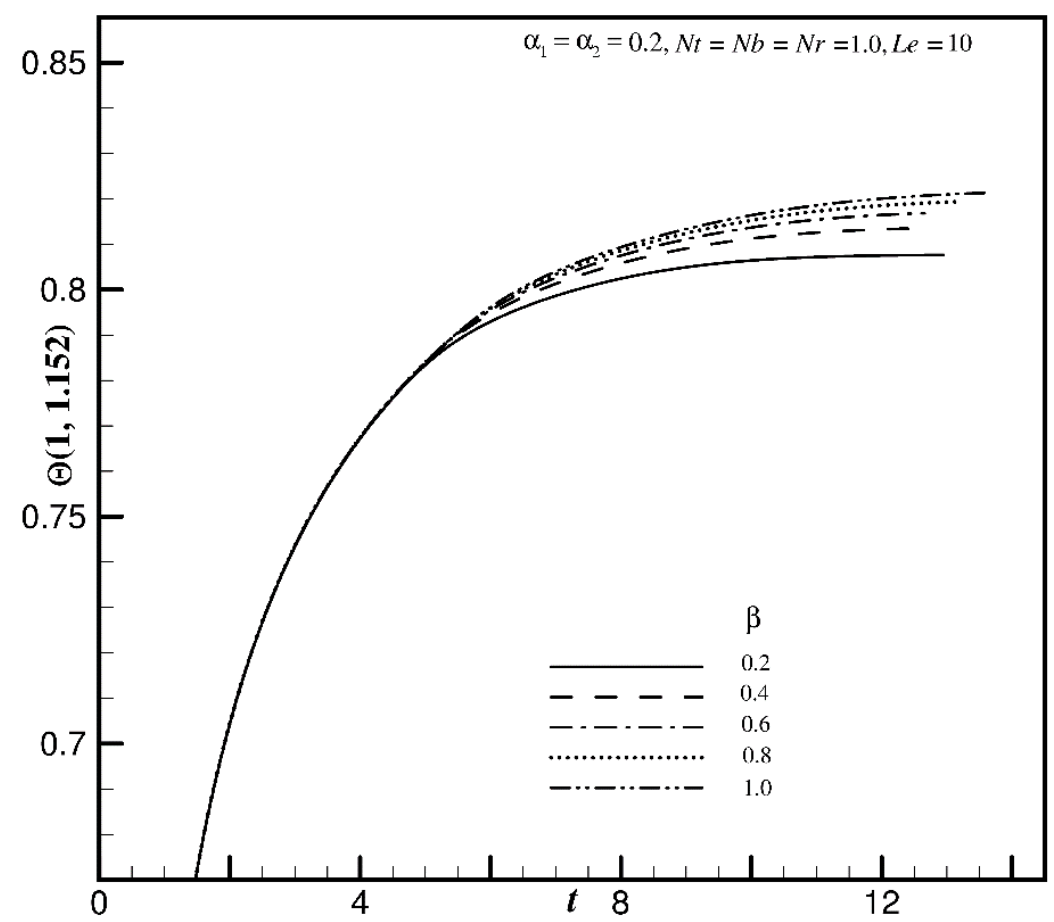

Fig. 6. Unsteady non-dimensional volume fraction profile against time $(t)$ for variation of third-grade fluid parameter. 


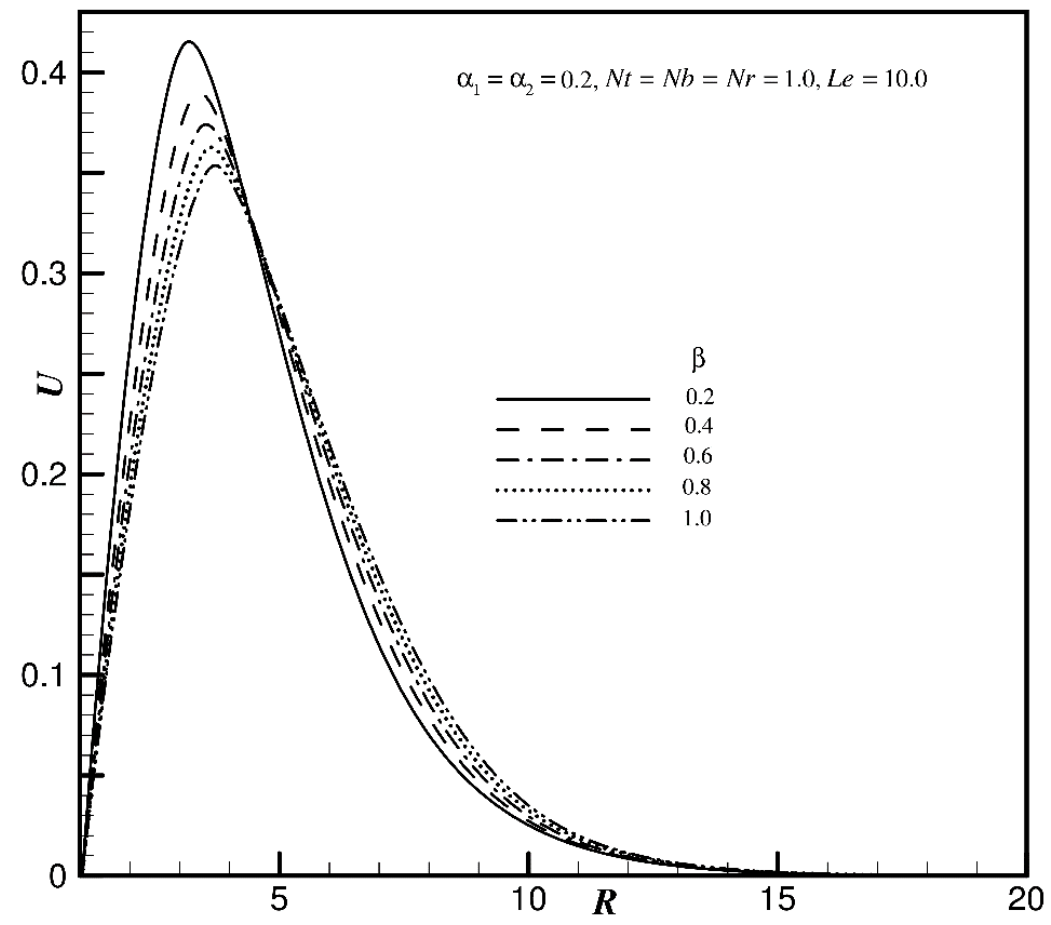

Fig. 7. Steady-state non-dimensional velocity profile against $R$ for variation of third-grade fluid parameter.

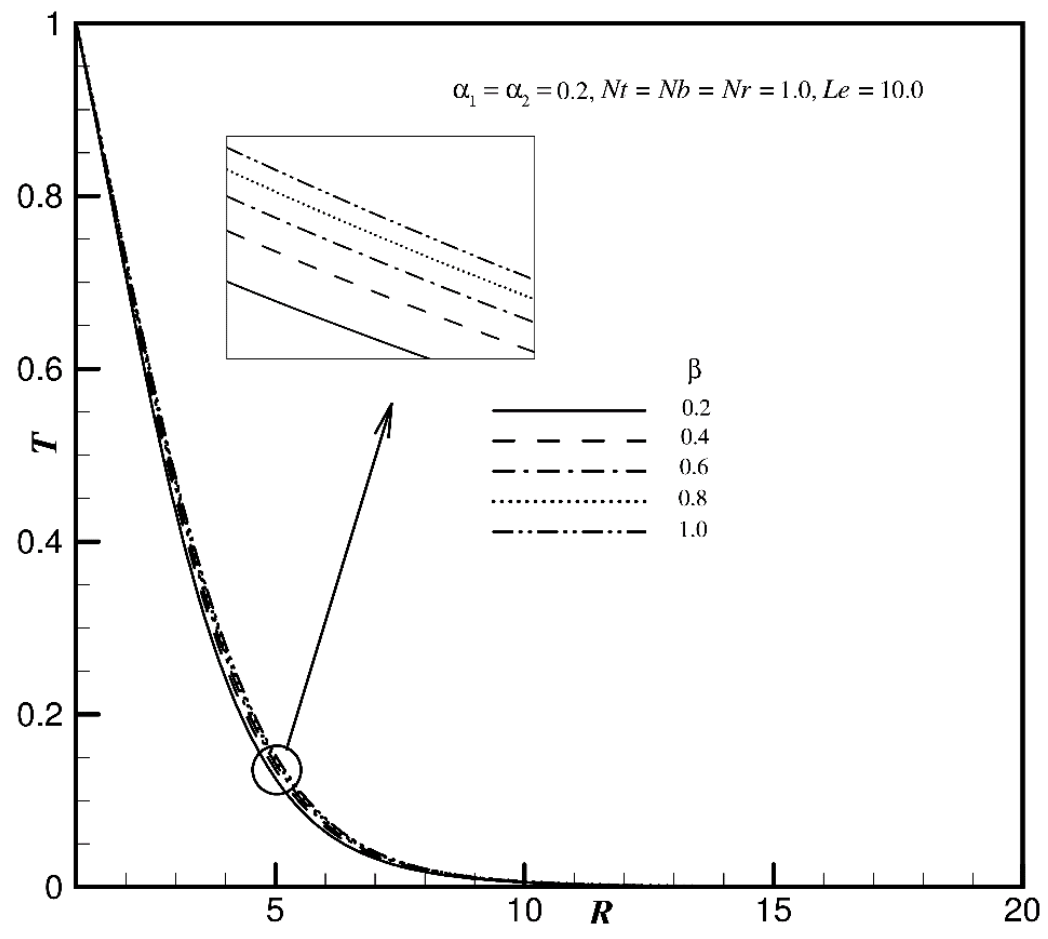

Fig. 8. Steady-state non-dimensional temperature profile against $R$ for variation of thirdgrade fluid parameter. 


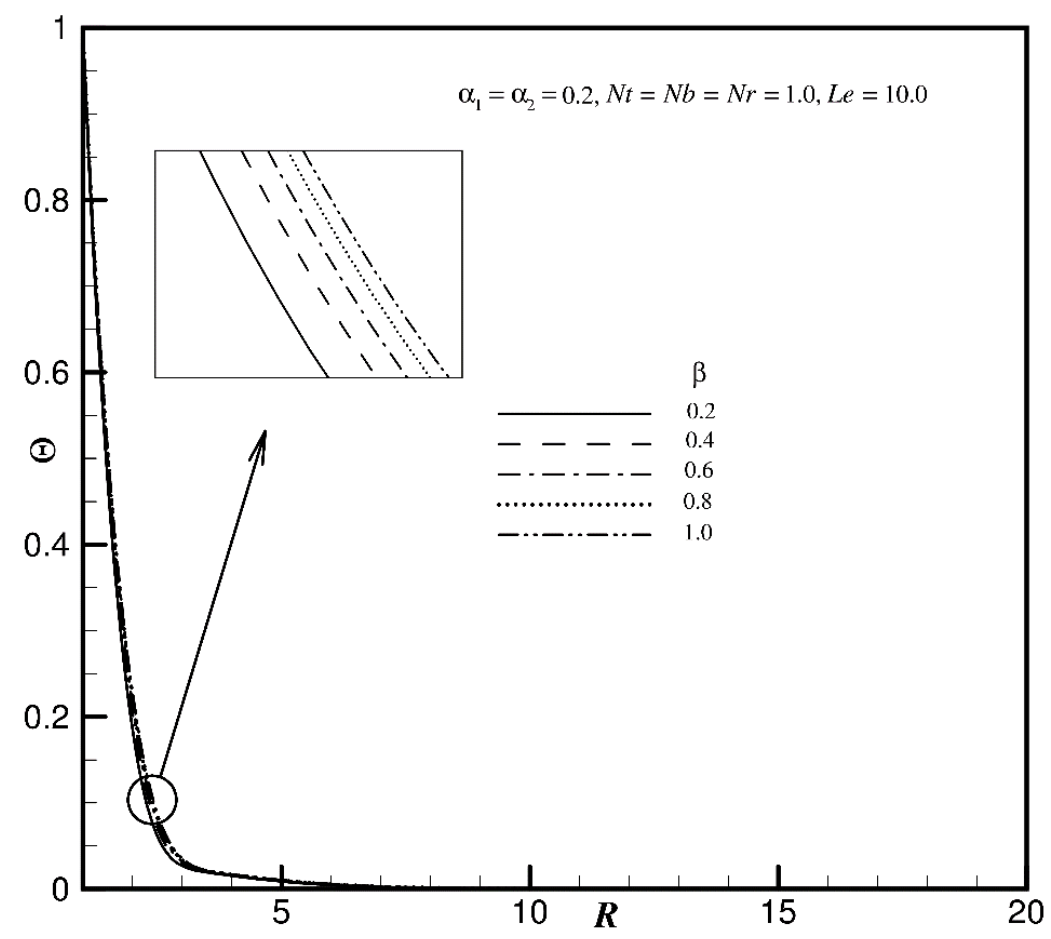

Fig. 9. Steady-state non-dimensional volume fraction profile against $R$ for variation of thirdgrade fluid parameter.

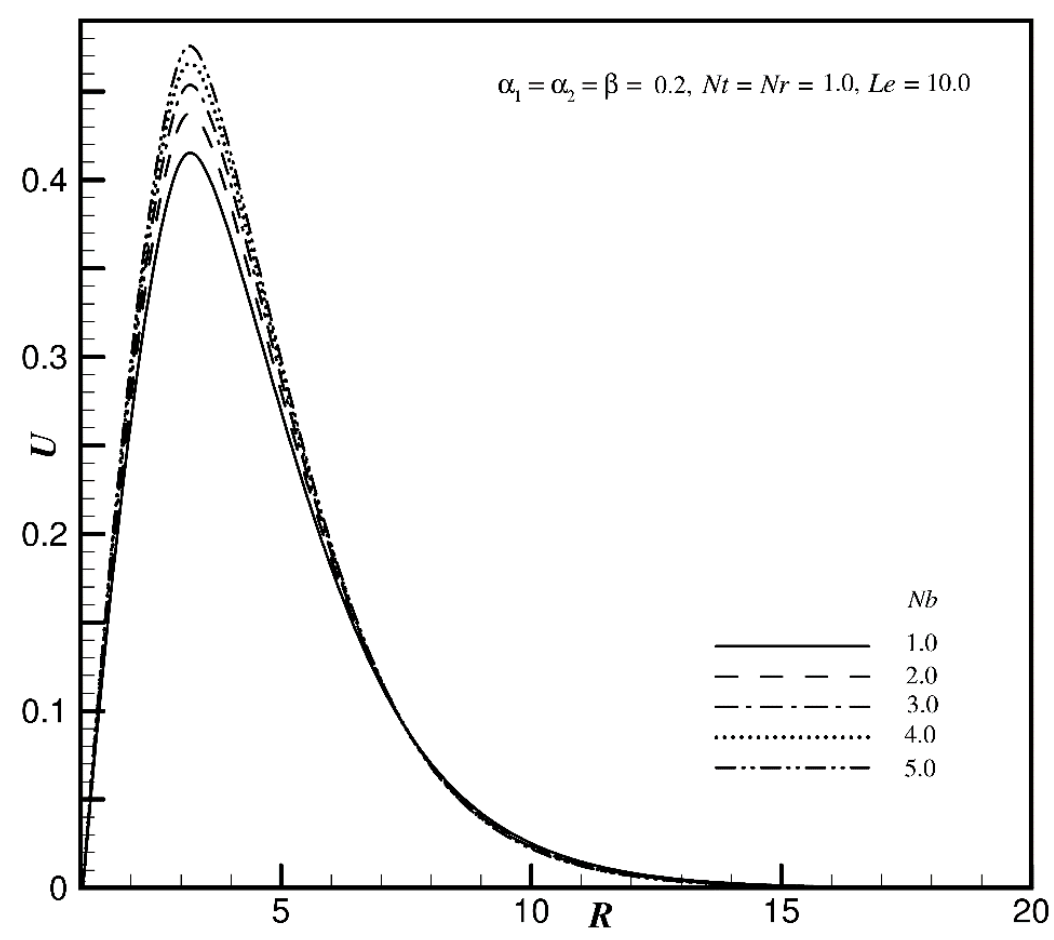

Fig. 10. Steady-state non-dimensional velocity profile against $R$ for variation of Brownian motion parameter. 


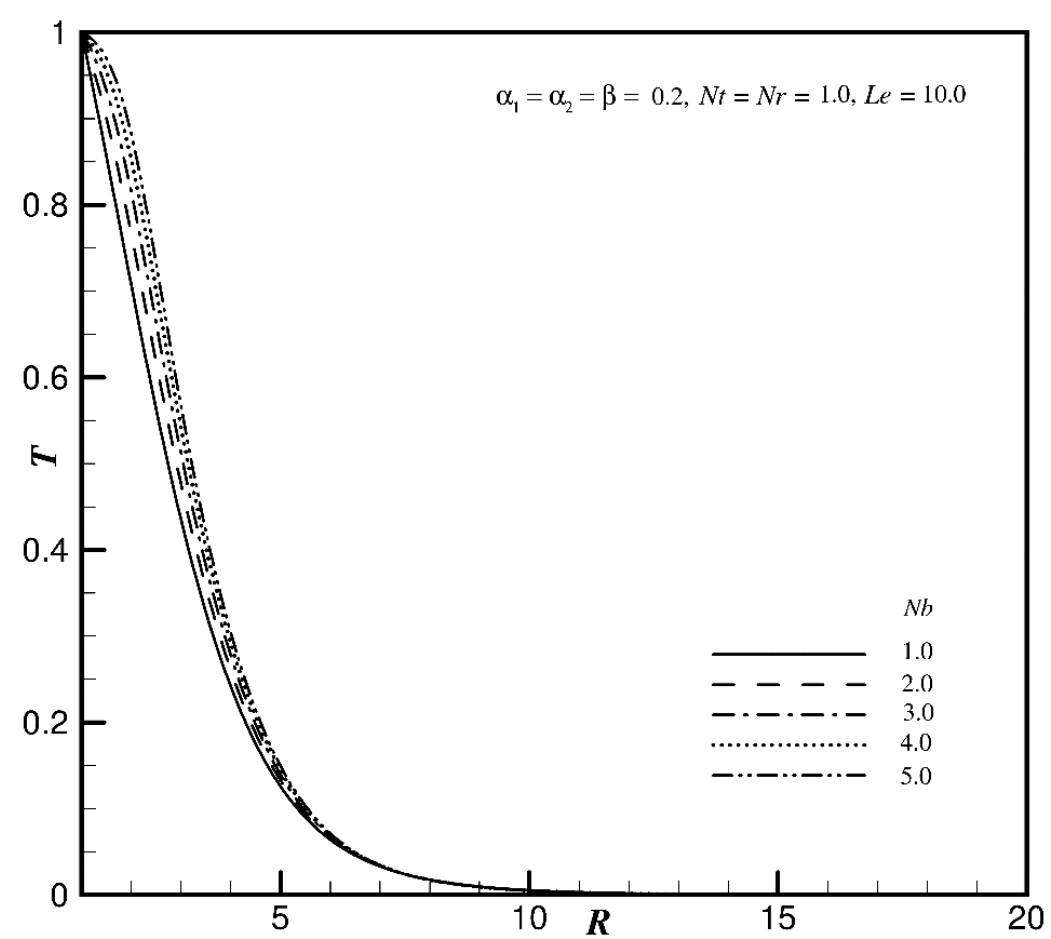

Fig. 11. Steady-state non-dimensional temperature profile against $R$ for variation of Brownian motion parameter.

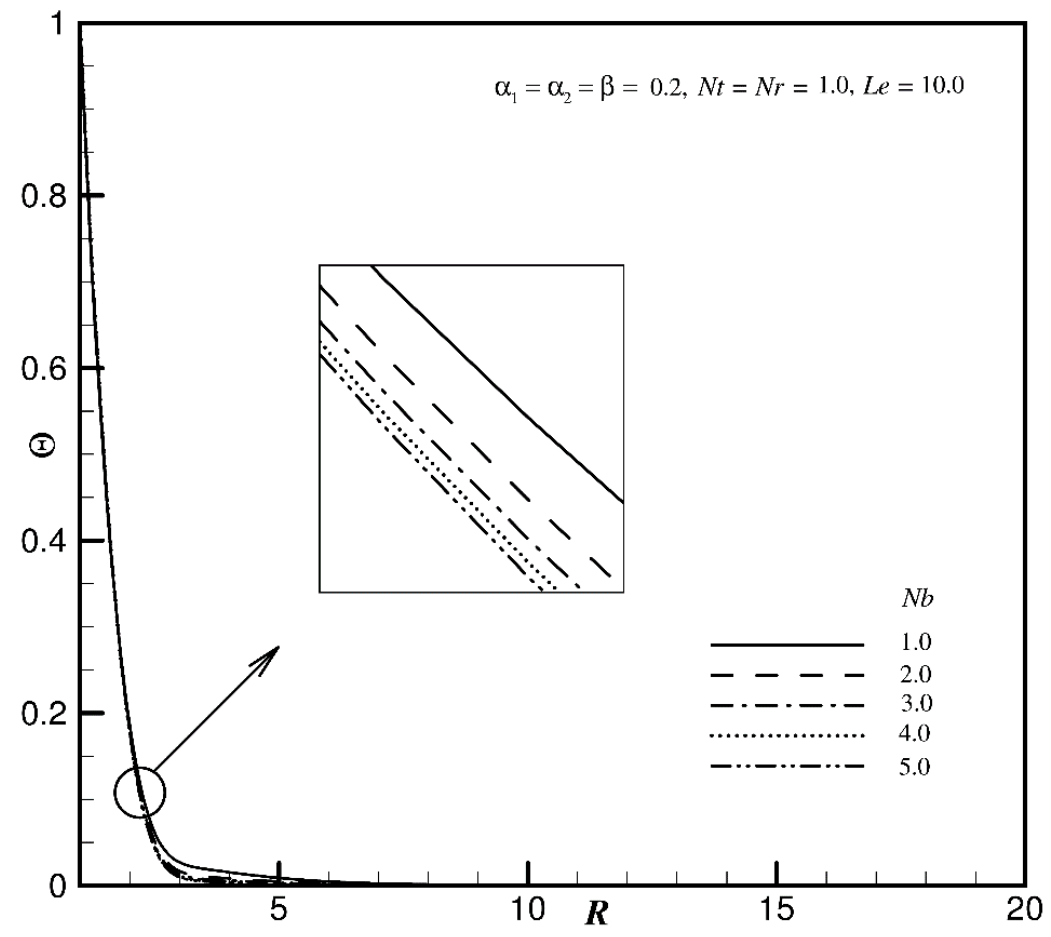

Fig. 12. Steady-state non-dimensional volume fraction profile against $R$ for variation of Brownian motion parameter. 


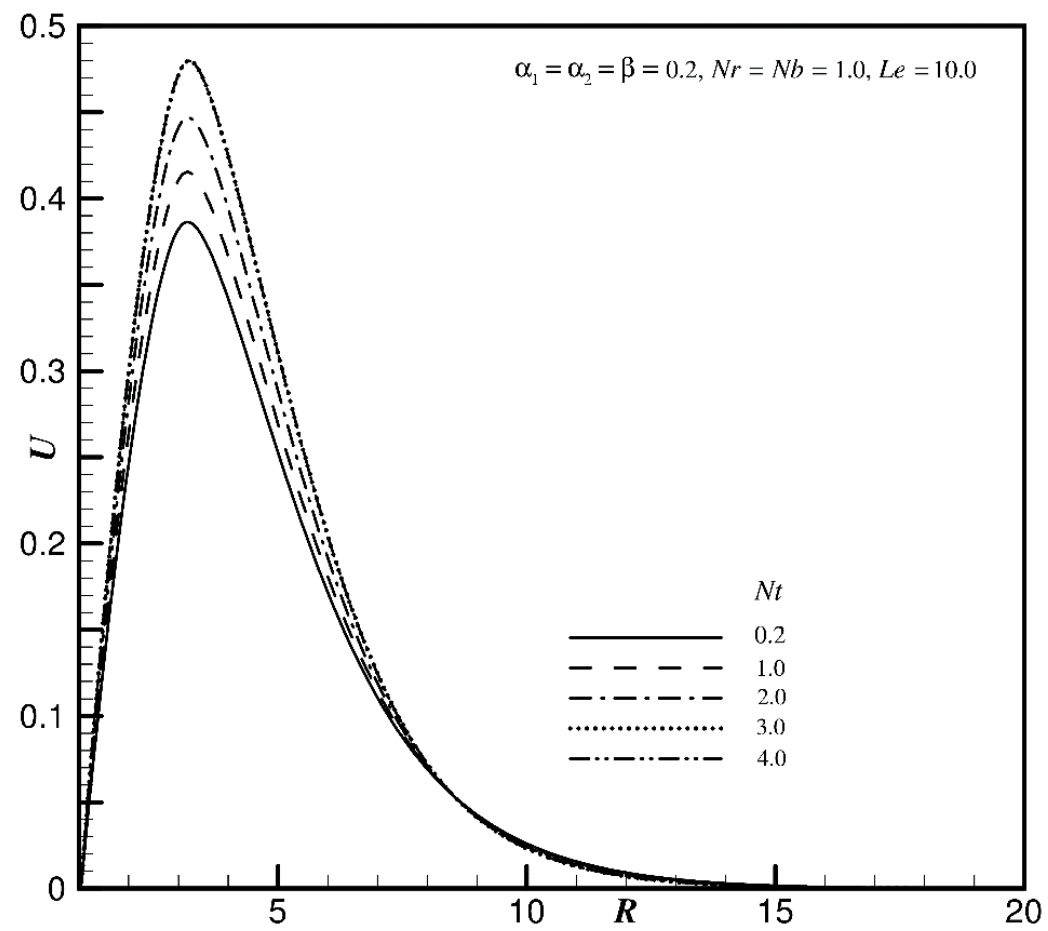

Fig. 13. Steady-state non-dimensional velocity profile against $R$ for variation of thermophoresis parameter.

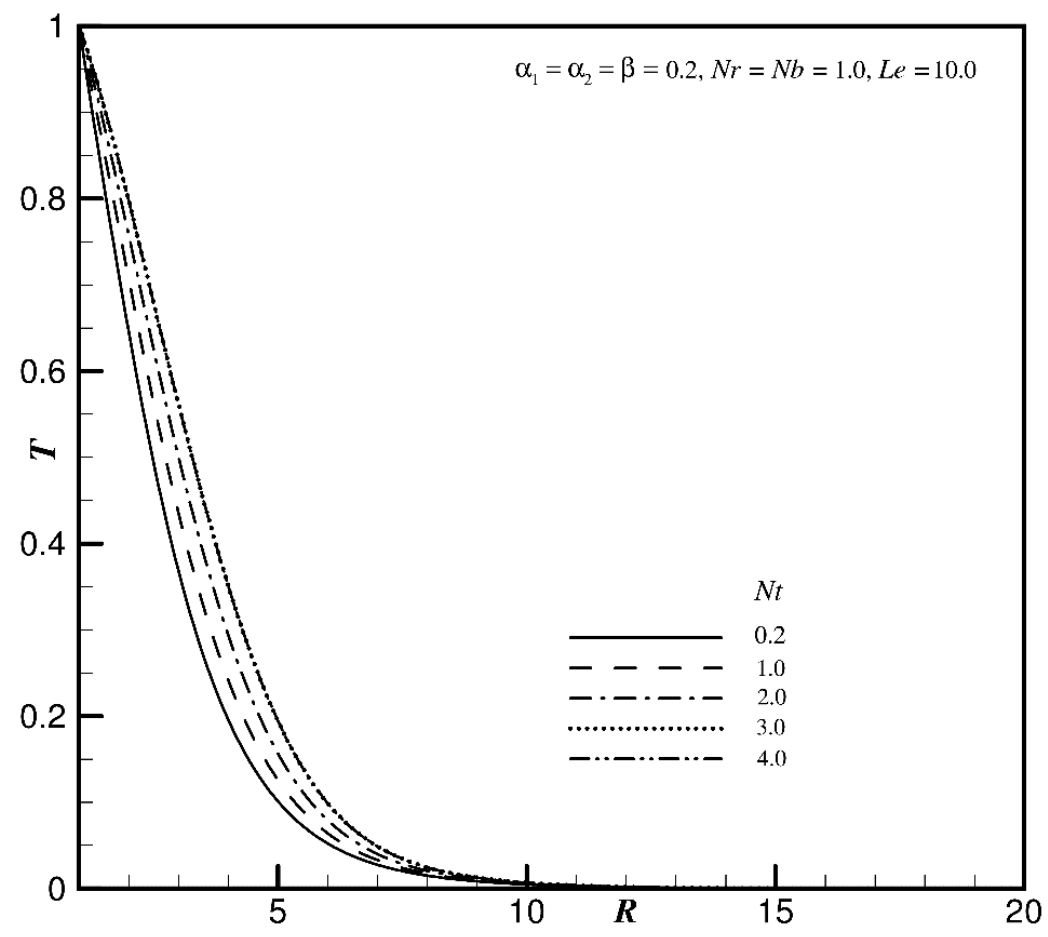

Fig. 14. Steady-state non-dimensional temperature profile against $R$ for variation of thermophoresis parameter. 


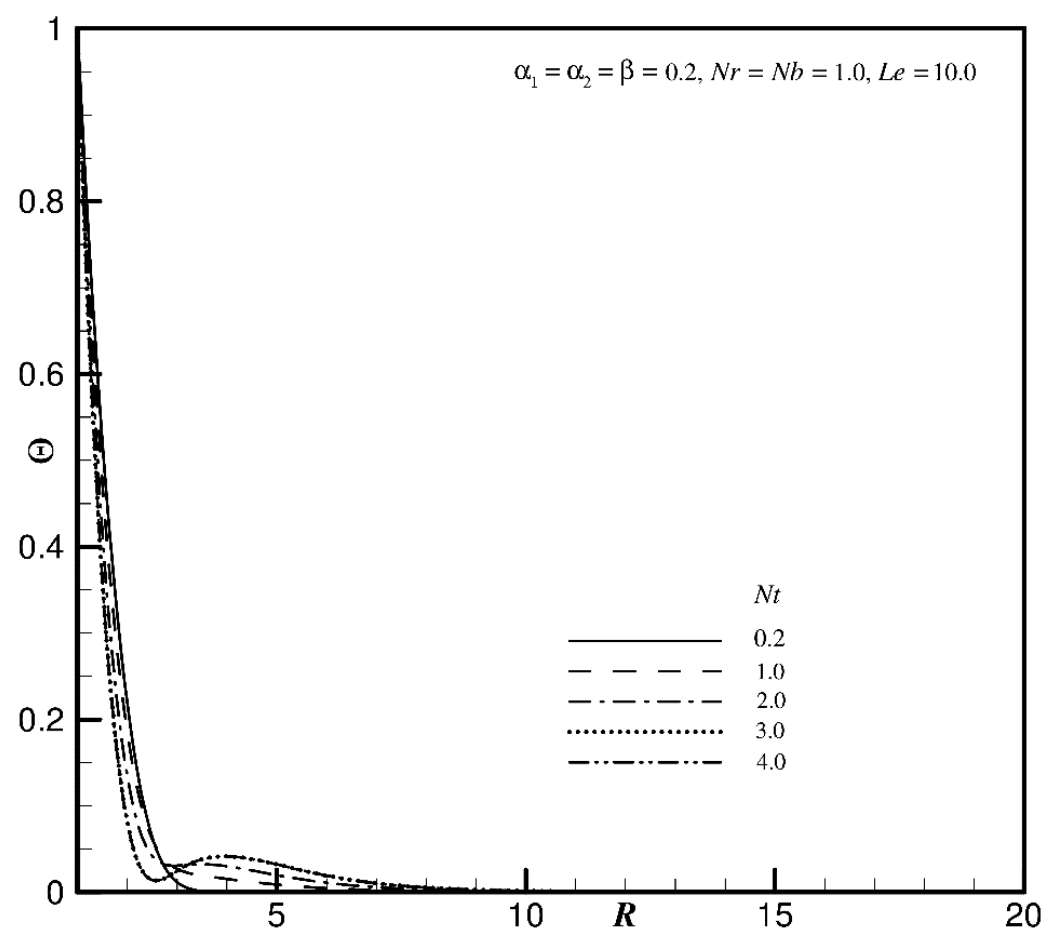

Fig. 15. Steady-state non-dimensional volume fraction profile against $R$ for variation of thermophoresis parameter.

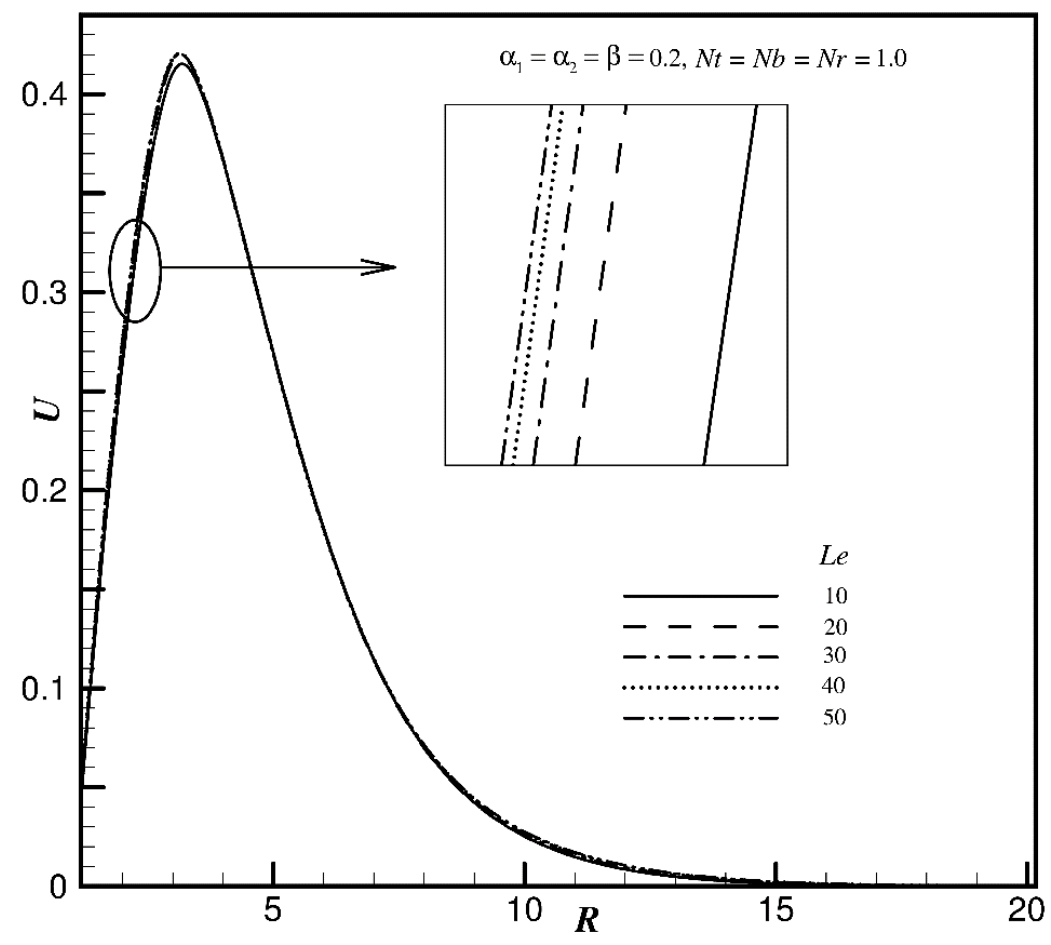

Fig. 16. Steady-state non-dimensional velocity profile against $R$ for variation of Lewis parameter. 


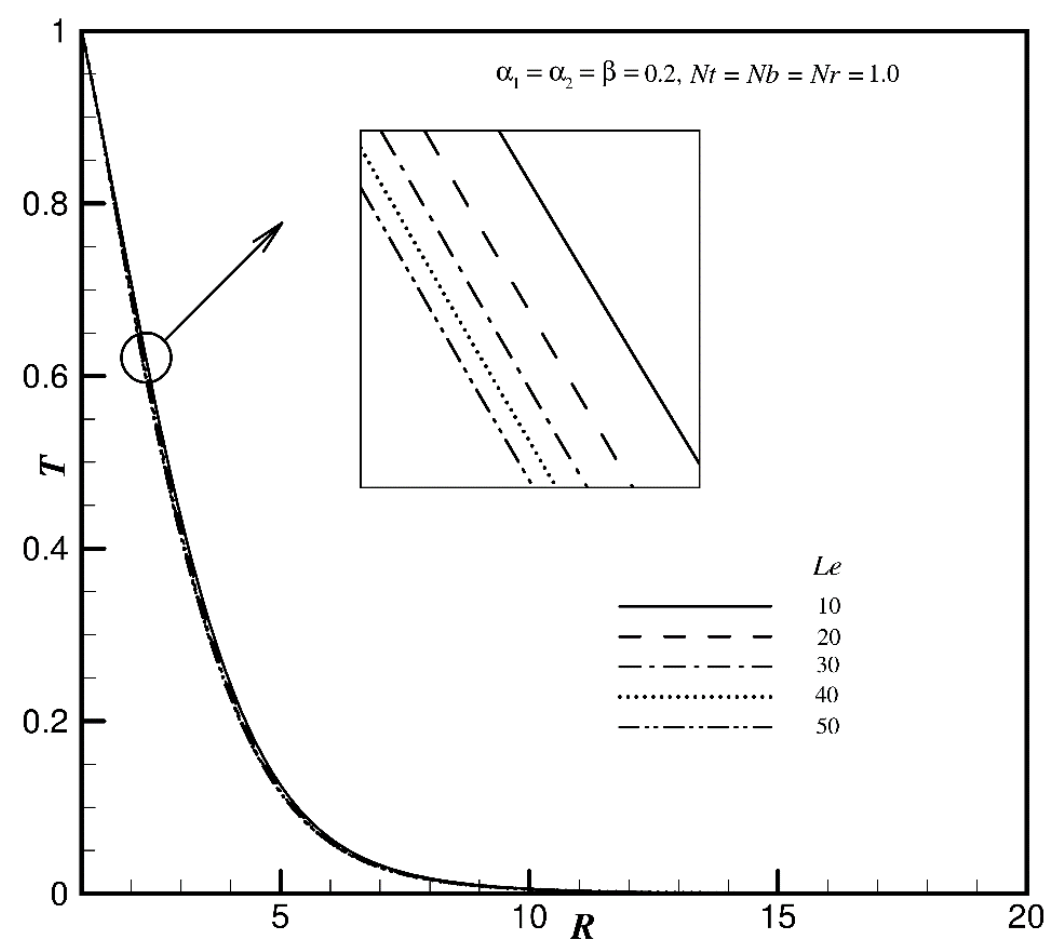

Fig. 17. Steady-state non-dimensional temperature profile against $R$ for variation of Lewis parameter.

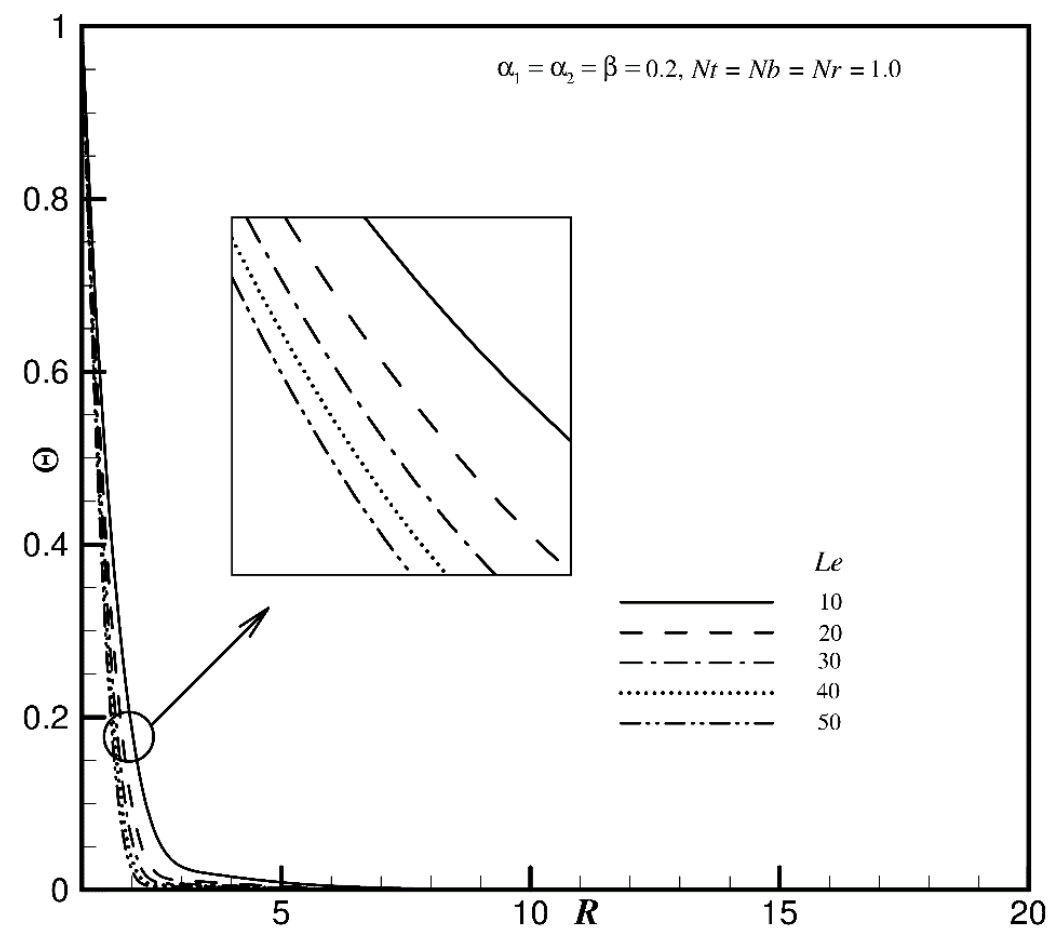

Fig. 18. Steady-state non-dimensional volume fraction profile against $R$ for variation of Lewis parameter. 


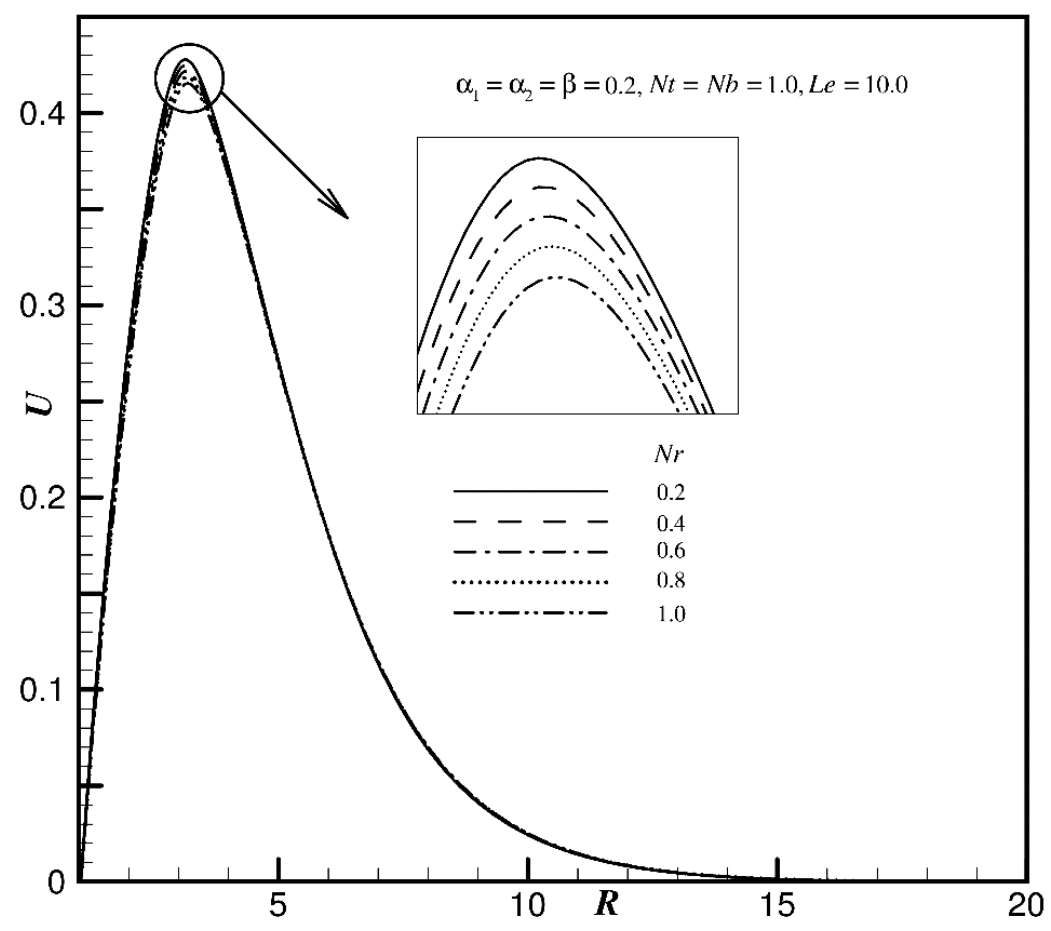

Fig. 19. Steady-state non-dimensional velocity profile against $R$ for variation of buoyancy ratio parameter.

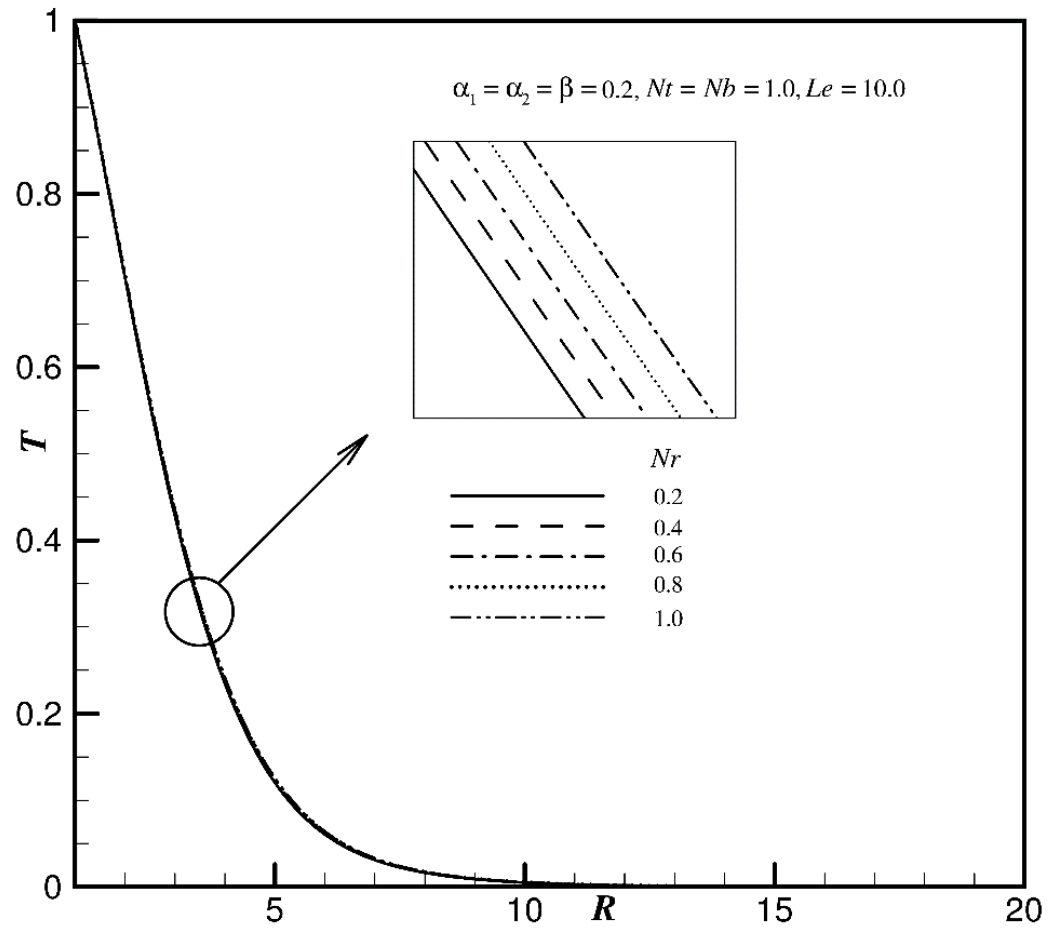

Fig. 20. Steady-state non-dimensional temperature profile against $R$ for variation of buoyancy ratio parameter. 


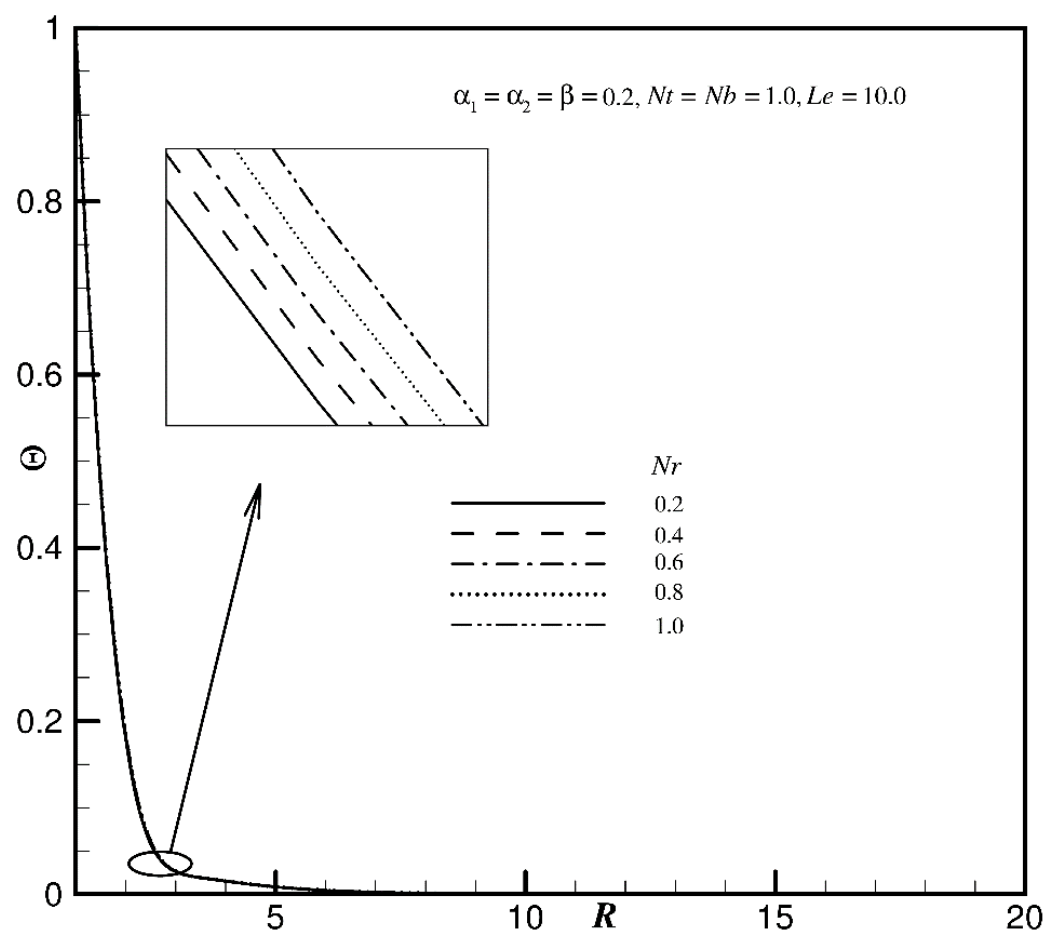

Fig. 21. Steady-state non-dimensional volume fraction profile against $R$ for variation of buoyancy ratio parameter.

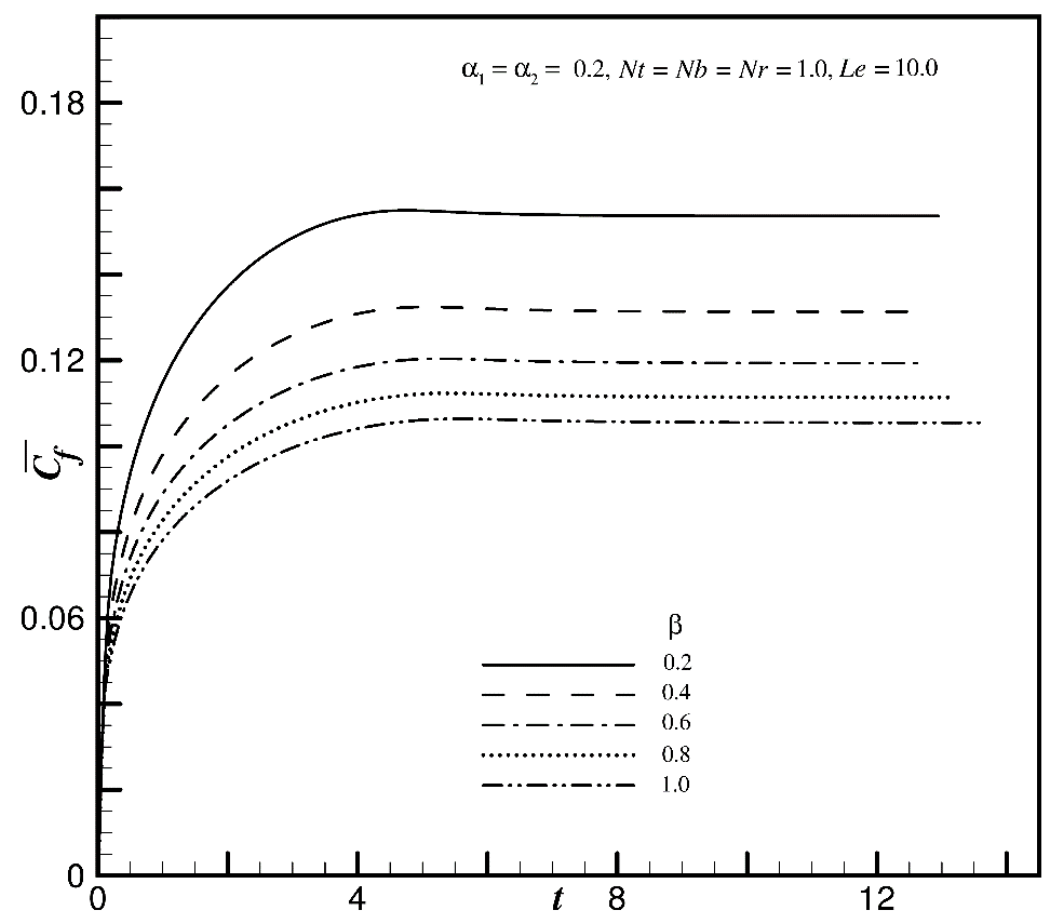

Fig. 22. Variation of third-grade fluid parameter on skin-friction against time $(t)$. 


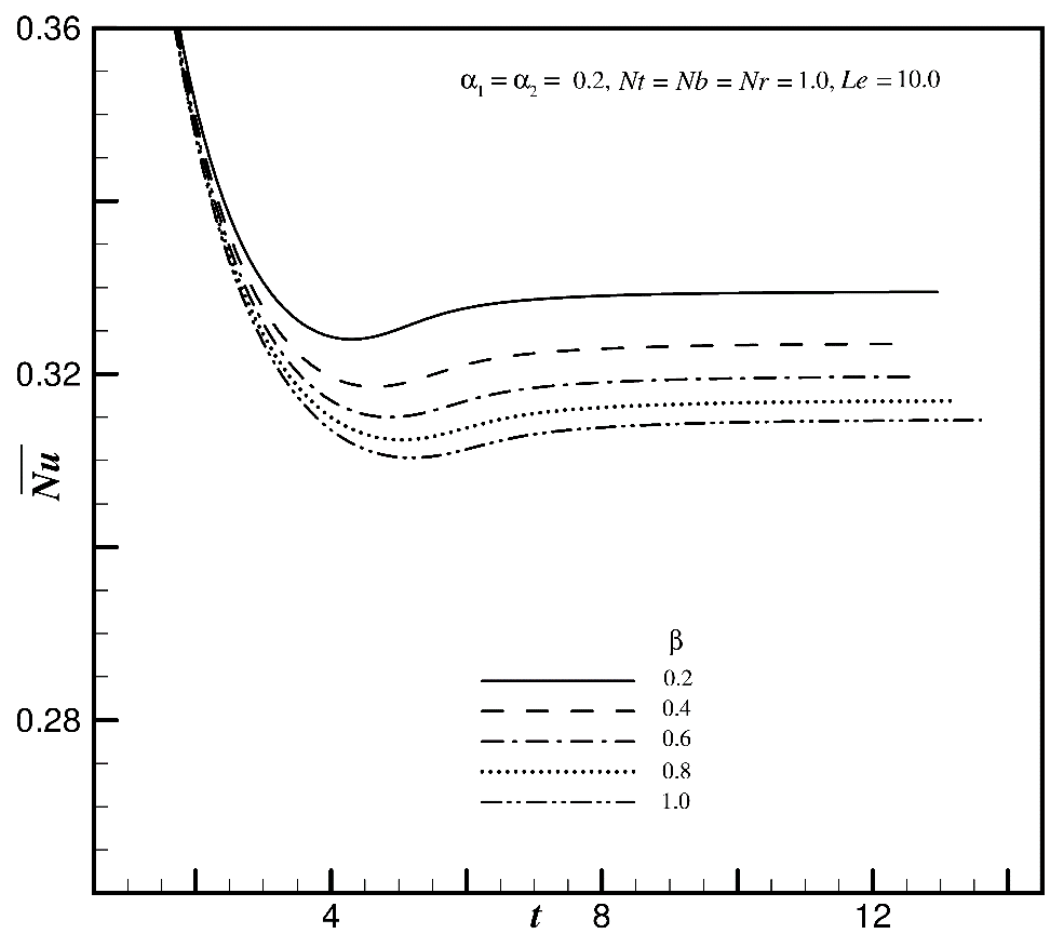

Fig. 23. Variation of third-grade fluid parameter on skin-friction against time $(t)$.

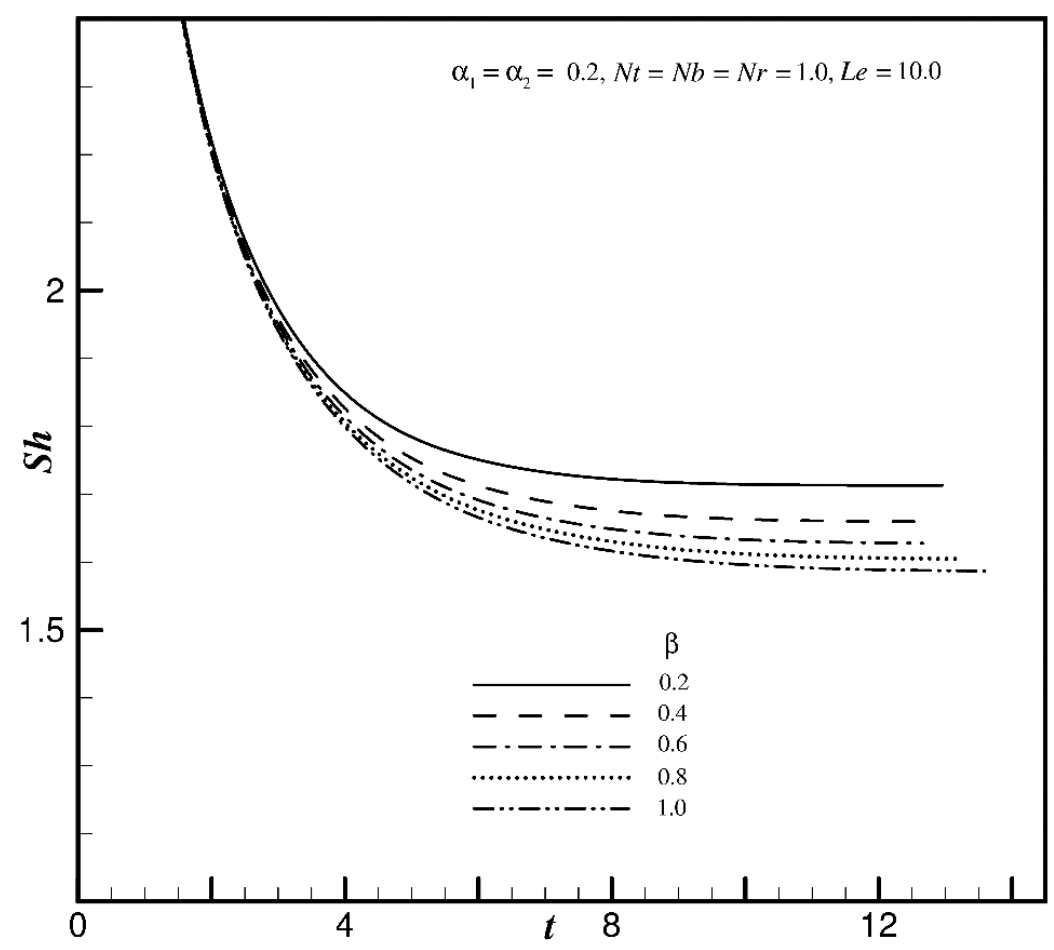

Fig. 24. Variation of third-grade fluid parameter on skin-friction against time $(t)$. 

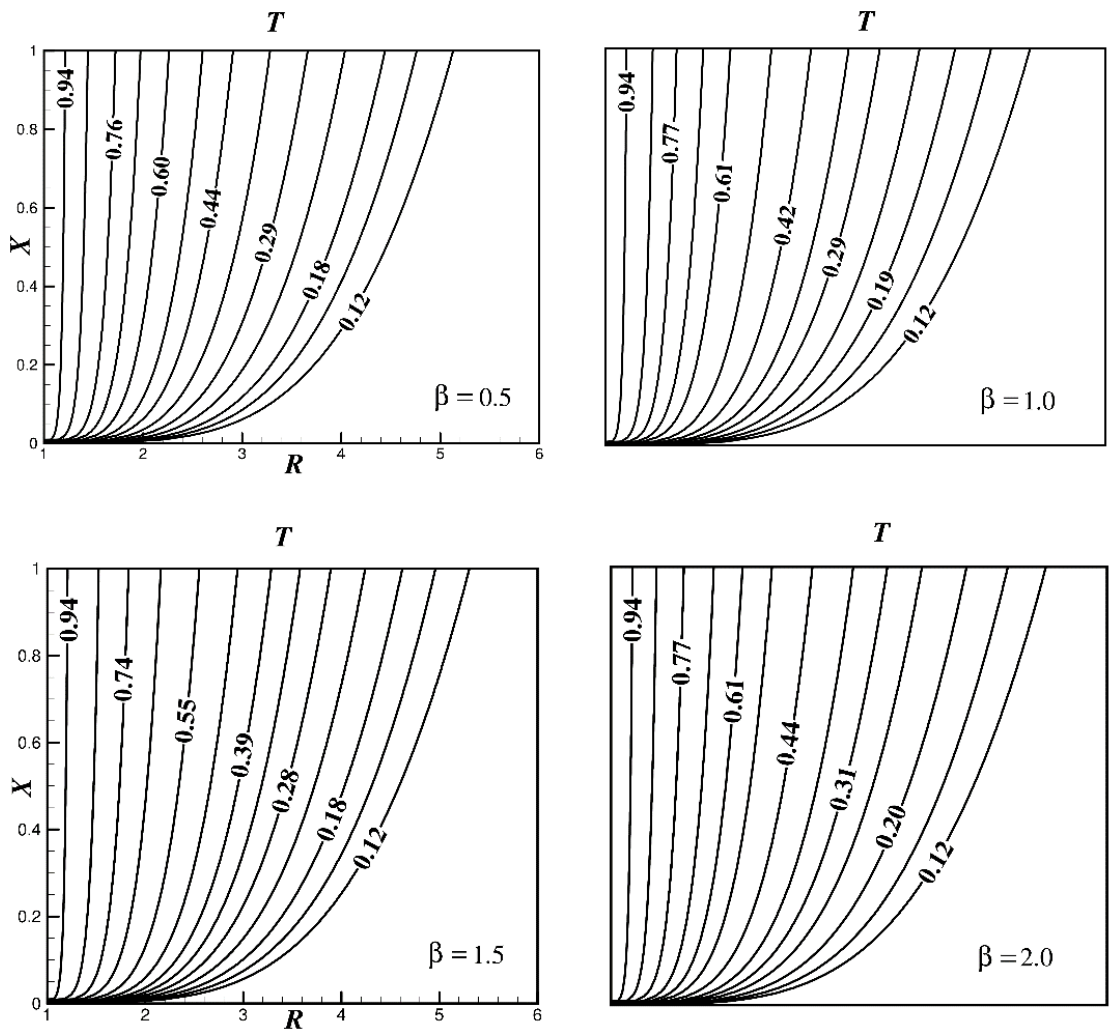

Fig. 25. Steady-state isotherms for different values of third-grade fluid parameter for fixed $\operatorname{Pr}=0.7$ and $G r=10.0$
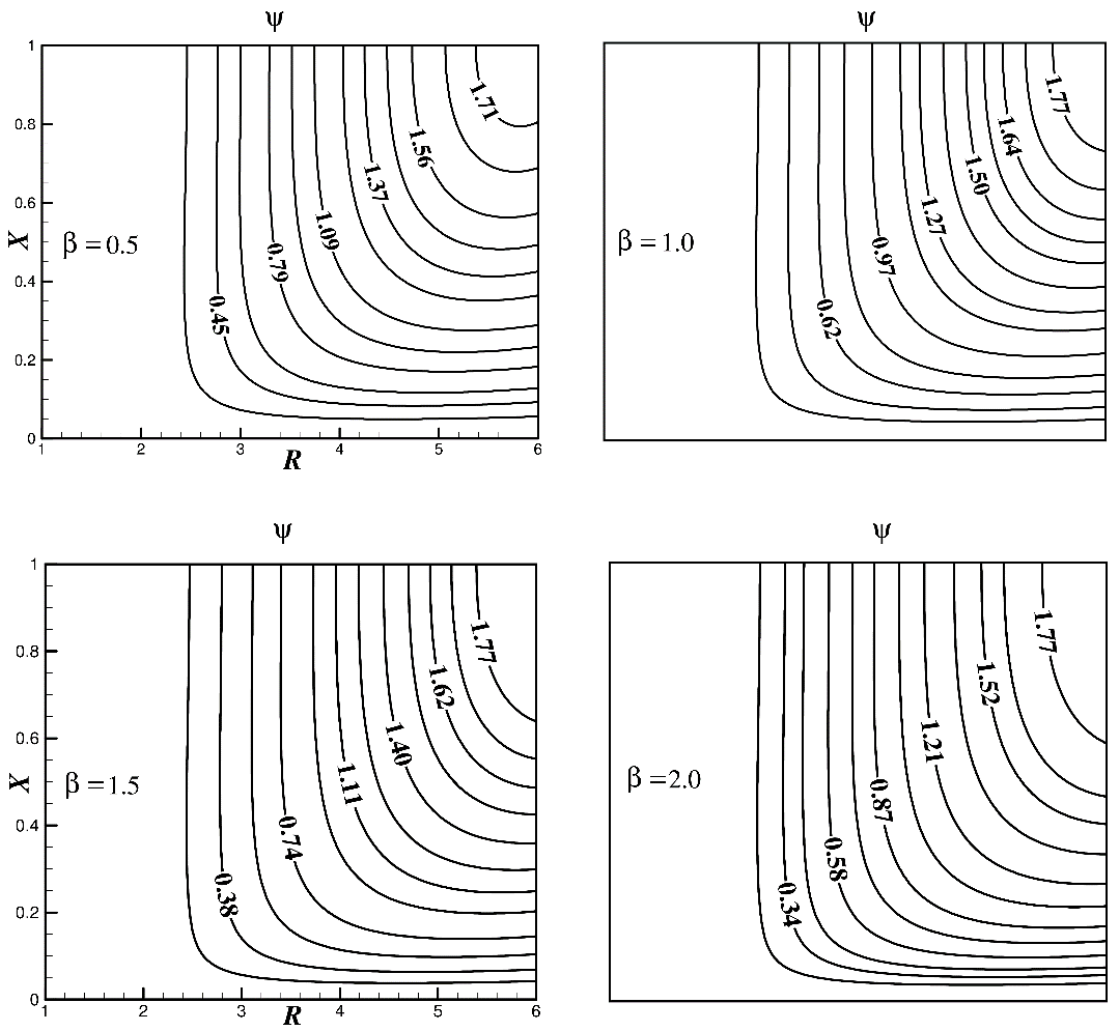

Fig. 26. Steady-state streamlines for different values of third-grade fluid parameter for fixed $\operatorname{Pr}=0.7$ and $G r=10.0$ 

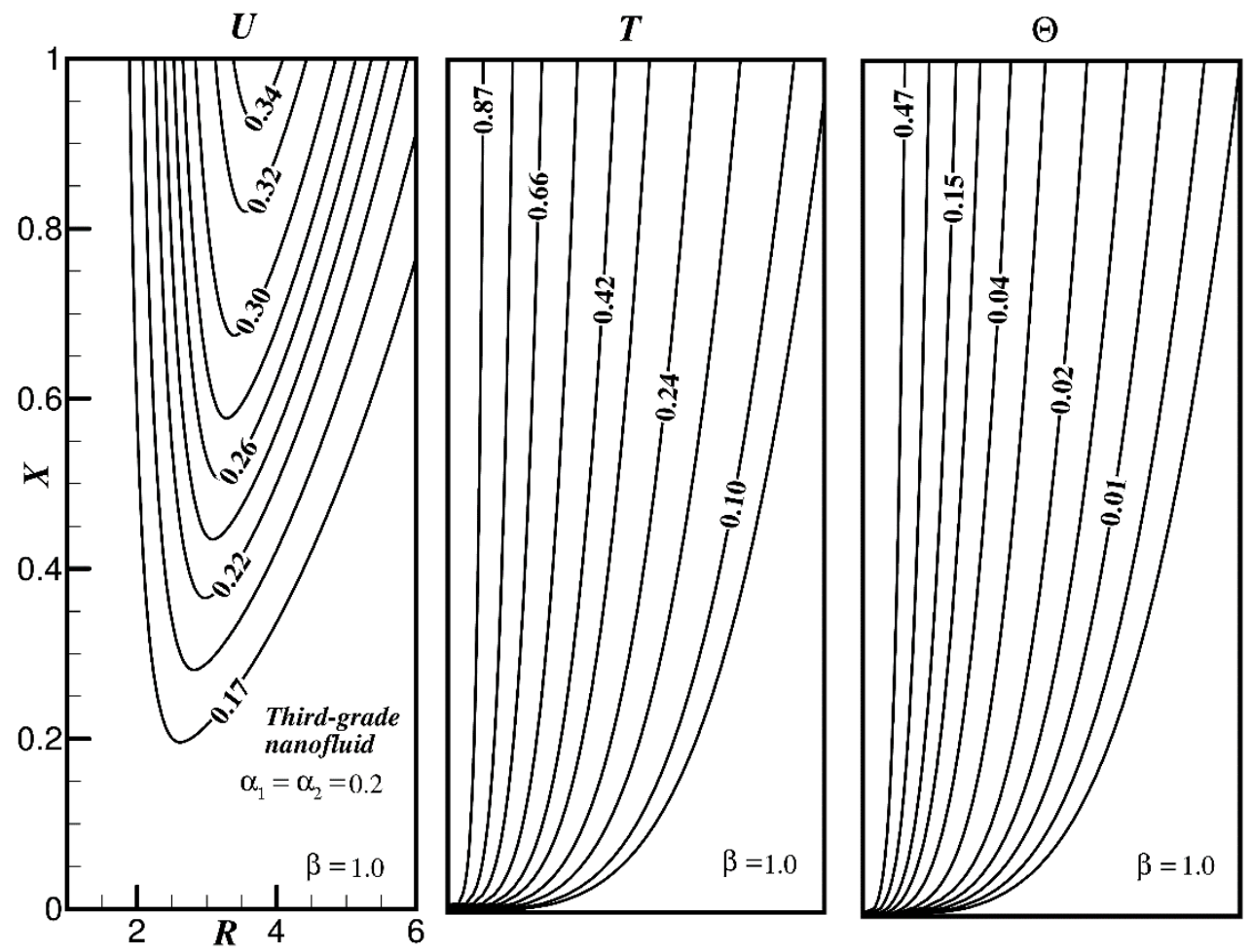

\section{7(a)}
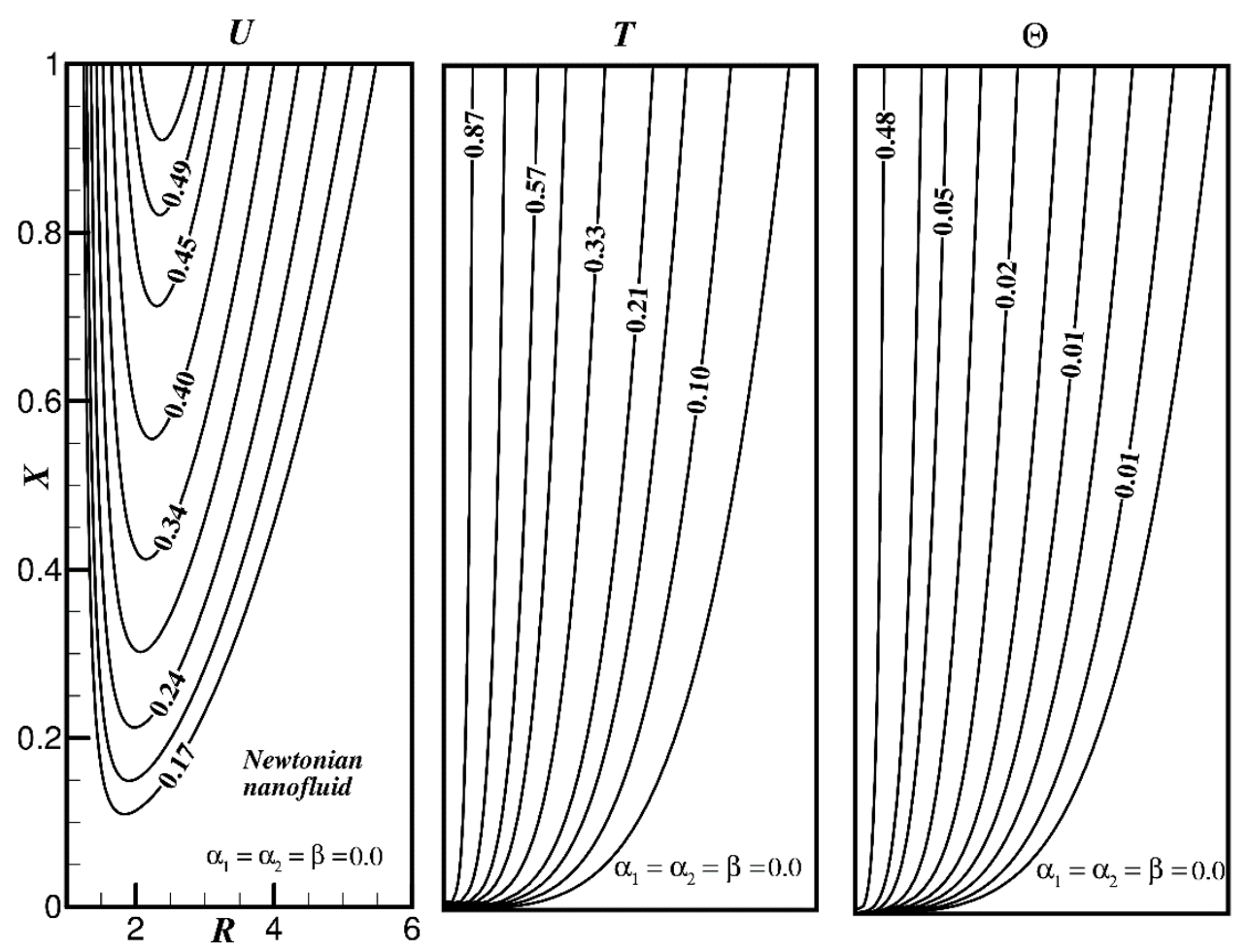

27(b)

Fig. 27. Steady-state contours of velocity $(U)$, temperature $(T)$ and volume fraction $(\Theta)$ of (a) third-grade nanofluid (b) Newtonian nanofluid for fixed $\mathrm{Pr}=0.7$ and $\mathrm{Gr}=10.0$ 


\section{REFERENCES}

[1] Y. Yang and Y. F. Cheng, Mechanistic aspects of electrodeposition of Ni-Co-SiC composite nano-coating on carbon steel, Electrochimica Acta, 109, 638-644 (2013).

[2] P.R. Dheeraj, Arghya Patra, Srijan sengupta, Siddhartha Das and Karabi Das, Synergistic effect of peak current density and nature of surfactant on microstructure, mechanical and electrochemical properties of pulsed electrodeposited Ni-Co-SiC nanocomposites, J. Alloys and Compounds, 729, 1093-1107 (2017).

[3] G. Song, Guoqiang Xu, Yongkai Quan, Qingchun Yuan and Philip A. Davies, Uniform design for the optimization of $\mathrm{Al}_{2} \mathrm{O}_{3}$ nanofilms produced by electrophoretic deposition, Surface and Coatings Technology, 286, 268-278 (2016).

[4] S.U.S. Choi, Enhancing thermal conductivity of fluids with nanoparticles, Developments and Applications of non-Newtonian flows (eds. Siginer, D. A. and Wang, H. P.), American Society of Mechanical Engineers (1995).

[5] H. Zhang, Hui Zhang, Longcheng Tang, Zhong Zhang, Lei Gu, Youzhong Xu and Christian Eger, Wear-resistant and transparent acrylate-based coating with highly filled nanosilica particles, Tribology International, 43, 83-91 (2010).

[6] S. Satish, B. Chandar Shekar and R. Sathyamoorthy, Nano polymer films by fast dip coating method for field effect transistor applications, Physics Procedia, 49, 166-176 (2013).

[7] H. Masuda, Ebata, A., Teramae, K., and Hishinuma, N. Alteration of thermal conductivity and viscosity of liquid by dispersing ultra-fine particles, Netsu Bussei, 7 (4), 227-233 (1993).

[8]Yimin Xuan and Qiang Li, Heat transfer enhancement of nanofluids, International Journal of Heat and Fluid Flow, 21 (1) 58-64 (2000).

[9] Yimin Xuan and Wilfried Roetzel, Conceptions for heat transfer correlation of nanofluids, International Journal of Heat and Mass Transfer, 43 (19), 3701-3707 (2000).

[10] Nandy Putra, Wilfried Roetzel and Sarit K. Das, Natural convection of nanofluids, Heat and Mass Transfer, 39 (8-9), 775-784 (2003).

[11] Elena V Timofeeva, Wenhua Yu, David M France, Dileep Singh and Jules L Routbort, Nanofluids for heat transfer: An engineering approach, Nanoscale Research Letters, 6-182 (2011).

[12] Wei Yu and Huaqing Xie, A review on nanofluids: preparation, stability mechanisms, and applications, J. Nanomaterials, 1-17 (2012).

[13] J. Buongiorno, Convective transport in nanofluids, ASME J. Heat Transfer, 128(3), 240250 (2006).

[14] R. K. Tiwari, M. K. Das, Heat transfer augmentation in a two-sided lid-driven differentially heated square cavity utilizing nanofluids, Int. J. Heat Mass Transf., 50 (9-10), 2002-2018 (2007) 
[15] O. Anwar Bég, Nonlinear multi-physical laminar nanofluid bioconvection flows: Models and computation, A. Sohail, Z. Li (Eds.): Computational Approaches in Biomedical NanoEngineering, Wiley, 113-145 (2018).

[16] Kuznetsov, A. V. and Nield, D. A. Natural convective boundary-layer flow of a nanofluid past a vertical plate, International Journal of Thermal Sciences, 49 (2), 243-247 (2010).

[17] M. A. Sheremet, I. Pop, A. Shenoy, Unsteady free convection in a porous open wavy cavity filled with a nanofluid using Buongiorno's mathematical model, International Communications in Heat and Mass Transfer, 67, 66-72 (2015).

[18] Azizah Mohd Rohni, Syakila Ahmad, Ahmad Izani Md. Ismail and Ioan Pop, Flow and heat transfer over an unsteady shrinking sheet with suction in a nanofluid using Buongiorno's model, International Communications in Heat and Mass Transfer, 43, 75-80 (2013)

[19] V. Rajesh, O. Anwar Bég and M. P. Mallesh, Transient nanofluid flow and heat transfer from a moving vertical cylinder in the presence of thermal radiation: Numerical study, Proc. IMECHE-Part N: J. Nanoengineering and Nanosystems, 230, 3-16 (2014).

[20] Muhammad Ijaz Khan, Siraj Ullah, Tasawar Hayata, Muhammad Waqas, Muhammad Imran Khan and Ahmed Alsaedi, Salient aspects of entropy generation optimization in mixed convection nanomaterial flow, International Journal of Heat and Mass Transfer, 126, 13371346 (2018).

[21] V.R. Prasad, S. A. Gaffar and O. Anwar Bég, Non-similar computational solutions for free convection boundary-layer flow of a nanofluid from an isothermal sphere in a non-Darcy porous medium, J. Nanofluids, 4, 203-213 (2015).

[22] O. Anwar Bég, F. Mabood and M. Nazrul Islam, Homotopy simulation of nonlinear unsteady rotating nanofluid flow from a spinning body, International Journal of Engineering Mathematics, 2015, 1-15 (2015).

[23] A. J. Chamkha, A. M. Rashad and A. M. Aly, Transient natural convection flow of a nanofluid over a vertical cylinder, Meccanica, 48 (1), 71-81 (2013).

[24] Yu Wang, Xiaojun Wu, Wei Yang, Yuanming Zhai, Banghu Xie and Mingbo Yang, Aggregate of nanoparticles: rheological and mechanical properties, Nanoscale Res Lett., 6- 114 (2011).

[25] Yuji Aoki, Akira Hatano and Hiroshi Watanabe, Rheology of carbon black suspensions. I. Three types of viscoelastic behaviour, Rheol Acta., 42 (3), 209-216 (2003).

[26] Fangming Du, Robert C. Scogna, Wei Zhou, Stijn Brand, John E. Fischer and Karen I. Winey, Nanotube networks in polymer nanocomposites: Rheology and electrical conductivity. Macromolecules, 37 (24), 9048-9055 (2004).

[27] L. Elias, F. Fenouillot, J. C. Majeste, P. Alcouffe, P. Cassagnau, Immiscible polymer blends stabilized with nano-silica particles: Rheology and effective interfacial tension, Polymer., 49 (20), 4378-4385 (2008). 
[28] H. Chang, C. S. Jwo, C. H. Lo, T. T. Tsung, M. J. Kao and H. M. Lin, Rheology of CuO nanoparticle suspension prepared by ASNSS, Rev. Adv. Mater. Sci., 10, 128-132 (2005).

[29] N. A. Latiff, M. J. Uddin, O. Anwar Bég and AI. M. Ismail, Unsteady forced bioconvection slip flow of a micropolar nanofluid from a stretching/ shrinking sheet, Proc. IMechE-Part N: J. Nanomaterials, Nanoengineering and Nanosystems, 230 (4), 177-187 (2016).

[30] T. Hayata, M. Z. Kiyani, A. Alsaedi, M. Ijaz Khan and I. Ahmad, Mixed convective threedimensional flow of Williamson nanofluid subject to chemical reaction, International Journal of Heat and Mass Transfer, 127, 422-429 (2018).

[31] M. J. Uddin, O. Anwar Bég, P. K. Ghose and A.I.M. Ismael, Numerical study of nonNewtonian nanofluid transport in a porous medium with multiple convective boundary conditions and nonlinear thermal radiation effects, Int. J. Num. Meth. Heat Fluid Flow, 26 (5) 1-25 (2016).

[32] P. Rana, R. Bhargava, O. Anwar Bég and A. Kadir, Finite element analysis of viscoelastic nanofluid flow with energy dissipation and internal heat source/sink effects, Int. J. Applied Computational Mathematics, 3, 1421-1447 (2017).

[33] C. H. Amanulla, N. Nagendra, M.S.N. Reddy, A.S. Rao and O. Anwar Bég, Mathematical study of non-Newtonian nanofluid transport phenomena from an isothermal sphere, Frontiers in Heat and Mass Transfer, 8, 29, 1-13 (2017).

[34] J. Prakash, E.P. Siva, D Tripathi, S. Kuharat and O. Anwar Bég, Peristaltic pumping of magnetic nanofluids with thermal radiation and temperature-dependent viscosity effects: modelling a solar magneto-biomimetic nanopump, Renewable Energy, 133, 1308-1326 (2019).

[35] R.L. Fosdick and K.R. Rajagopal, Thermodynamics and stability of fluids of third grade, Proc. Royal Society of London. Series A, 369, 351-377 (1980).

[36] R. Byron Bird, Robert C. Armstrong, Ole Hassager, Dynamics of Polymeric Liquids, Volume 1: Fluid Mechanics, ${ }^{\text {nd }}$ Edition, Wiley, USA (1987).

[37] T. Javed and I. Mustafa, Slip effects on a mixed convection flow of a third-grade fluid near the orthogonal stagnation point on a vertical surface, J. Applied Mechanics and Technical Physics, 57, 527-536 (2016).

[38] O. Anwar Bég, H. S. Takhar, R. Bhargava, S. Rawat, and V. R. Prasad, Numerical study of heat transfer of a third-grade viscoelastic fluid in a non-Darcy porous media with thermophysical effects, Physica Scripta, 77, 065402-13 (2008).

[39] B. Sahoo, Hiemenz flow and heat transfer of a third grade fluid, Communications in Nonlinear Science and Numerical Simulation, 14 (3), 811-826 (2009).

[40] B. Sahoo and Y. Do, Effect of slip on sheet-driven flow and heat transfer of a third grade fluid past a stretching sheet, Int. Comm. Heat and Mass Transfer, 37 (8), 1064-1071 (2010). 
[41] Y. M. Aiyesimi, M. Jiya, R. O. Olayiwola, A. A. Wachin, Mathematical analysis of convective flow of an unsteady magnetohydrodynamic (MHD) third grade fluid in a cylindrical channel, American J. Computational and Applied Mathematics, 6 (2), 103-108 (2016).

[42] G. J. Reddy, A. Hiremath, and M. Kumar, Computational modeling of unsteady thirdgrade fluid flow over a vertical cylinder: a study of heat transfer visualization, Results in Physics, 8, 671-682 (2018).

[43] G. J. Reddy, A. Hiremath, Hussain Basha and N. S. Venkata Narayanan, Transient flow and heat transfer characteristics of non-Newtonian supercritical third-grade fluid $\left(\mathrm{CO}_{2}\right)$ past a vertical cylinder, International Journal of Chemical Reactor Engineering, 16(8), 1542-6580 20170232 (2018).

[44] S. Abdul Gaffar, V. Ramachandra Prasad, O. Anwar Bég, Md. H. Hidayathullah Khan and $\mathrm{K}$. Venkatadri, Radiative and magnetohydrodynamics flow of third grade viscoelastic fluid past an isothermal inverted cone in the presence of heat generation/absorption, J. Brazilian Soc. Mech Sci. Eng,40, 127-146 (2018).

[45] U. Farooq, T. Hayat, A. Alsaedi and S. Liao, Heat and mass transfer of two-layer flows of third-grade nano-fluids in a vertical channel, Applied Math. Comp., 242, 528-540 (2014).

[46] S. Nadeem and S. Saleem, Analytical study of third grade fluid over a rotating vertical cone in the presence of nanoparticles, Int. J. Heat Mass Transfer, 85, 1041-1048 (2015).

[47] W. A. Khan, J. R. Culham and O. D. Makinde, Combined heat and mass transfer of thirdgrade nanofluids over a convectively-heated stretching permeable surface, The Canadian Journal of Chemical Engineering, 93, 1880-1888 (2015).

[48] S. Qayyum, T. Hayat and A. Alsaedi, Thermal radiation and heat generation/absorption aspects in third grade magneto-nanofluid over a slendering stretching sheet with Newtonian conditions, Physica B: Condensed Matter, 537, 139-149 (2018).

[49] T. Hayat, S. Ahmad, M. Ijaz Khan and A. Alsaedi, Modeling and analyzing flow of third grade nanofluid due to rotating stretchable disk with chemical reaction and heat source, Physica B: Condensed Matter, 537, 116-126 (2018).

[50] Dunn, J. E. and Rajagopal, K. R. Fluids of differential type: critical review and thermodynamic analysis, International Journal of Engineering Science, 33, 689 - 729 (1995).

[51] R. L. Fosdick and B. Straughan, Catastrophic instabilities and related results in a fluid of third grade. Int. J. Non-Linear Mech., 16, 191 (1981).

[52] B. Sahoo and S. Poncet, Flow and Heat Transfer of a third-grade fluid past an exponentially stretching sheet with partial slip boundary condition, International Journal of Heat and Mass Transfer, 54(23-24), 5010-5019 (2011).

[53] M. Keimanesh, M. M. Rashidi, A. J. Chamkha and R. Jafari, Study of a third-grade nonNewtonian fluid flow between two parallel plates using the multi-step deferential transform method, Computers \& Mathematics with Applications, 62 (8) 2871-2891 (2011). 
[54] M. Sajid, T. Hayat and S. Asghar, Non-similar analytic solution for MHD flow and heat transfer in a third-order fluid over a stretching sheet, International Journal of Heat and Mass Transfer, 50, 1723-1736 (2007).

[55] Hayat T, Nazar H, Imtiaz M, Alsaedi A, Ayub M. Axisymmetric squeezing flow of third grade fluid in presence of convective conditions, Chin J Phys., 55, 738-54 (2017).

[56] Hayat T, Mustafa M, Asghar S. Unsteady flow with heat and mass transfer of a third grade fluid over a stretching surface in the presence of chemical reaction, Nonlinear Anal: Real World Appl., 11, 3186-97 (2010).

[57] Carnahan B, Luther H A, Wilkes J O, Applied Numerical Methods. Wiley, USA (1969).

[58] Von Rosenberg DU, Methods for the Numerical Solution of Partial Differential Equations. American Elsevier Publishing Company, New York (1969).

[59] H. P. Rani and C. N. Kim, A numerical study on unsteady natural convection of air with variable viscosity over an isothermal vertical cylinder, Korean J. Chem. Eng., 27(3), 759-765 (2010).

[60] B. Vasu, R. S. R. Gorla, O. Anwar Bég, P. V. S. N. Murthy, V. R. Prasad and A. Kadir, Unsteady flow of a nanofluid over a sphere with non-linear Boussinesq approximation, AIAA J. Thermophysics and Heat Transfer, 33(2), 343-355 (2018). 